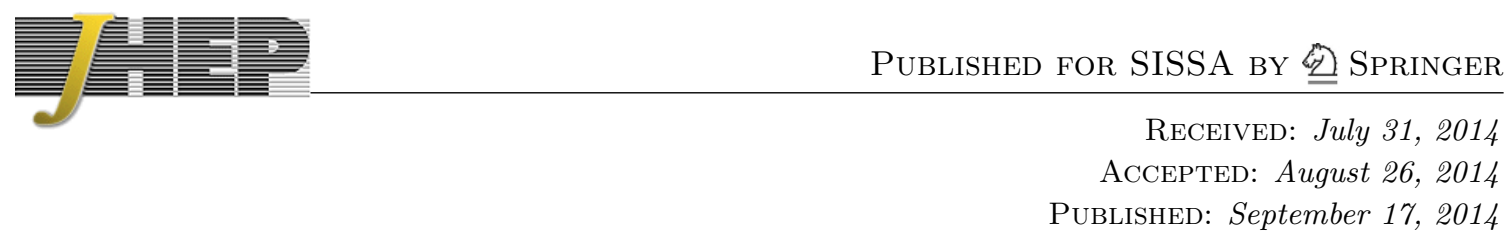

\title{
Higgs mass and unnatural supersymmetry
}

\author{
Emanuele Bagnaschi, ${ }^{a, b}$ Gian F. Giudice, ${ }^{c}$ Pietro Slavich ${ }^{a, b}$ and Alessandro Strumia ${ }^{d, e}$ \\ ${ }^{a}$ LPTHE, UPMC Paris 6, Sorbonne Universités, \\ 4 Place Jussieu, F-75252 Paris, France \\ ${ }^{b}$ LPTHE, CNRS, \\ 4 Place Jussieu, F-75252 Paris, France \\ ${ }^{c}$ CERN, Theory Division, \\ CH-1211 Geneva 23, Switzerland \\ ${ }^{d}$ Dipartimento di Fisica dell'Università di Pisa and INFN, \\ Largo B. Pontecorvo 3, I-56127 Pisa, Italy \\ ${ }^{e}$ National Institute of Chemical Physics and Biophysics, \\ Akadeemia tee 23, 12618 Tallinn, Estonia \\ E-mail: bagnaschi@lpthe.jussieu.fr, gian.giudice@cern.ch, \\ slavich@lpthe.jussieu.fr, Alessandro.Strumia@mail.df.unipi.it
}

ABSTRACT: Assuming that supersymmetry exists well above the weak scale, we derive the full one-loop matching conditions between the SM and the supersymmetric theory, allowing for the possibility of an intermediate Split-SUSY scale. We also compute two-loop QCD corrections to the matching condition of the Higgs quartic coupling. These results are used to improve the calculation of the Higgs mass in models with high-scale supersymmetry or split supersymmetry, reducing the theoretical uncertainty. We explore the phenomenology of a mini-split scenario with gaugino masses determined by anomaly mediation. Depending on the value of the higgsino mass, the theory predicts a variety of novel possibilities for the dark-matter particle.

Keywords: Higgs Physics, Beyond Standard Model, Supersymmetric Standard Model

ARXIV EPRINT: 1407.4081 


\section{Contents}

1 Introduction $\quad 1$

2 Threshold corrections from heavy superparticles 2

2.1 Lagrangian and tree-level matching 2

2.2 One-loop matching 4

2.3 Two-loop SUSY-QCD correction to the quartic Higgs coupling 10

3 The Higgs mass and supersymmetry 13

$\begin{array}{ll}3.1 & \text { Quasi-natural SUSY } \\ 3.2 & 13\end{array}$

$\begin{array}{ll}3.2 & \text { High-Scale SUSY } \\ 3.3 & \text { Split SUSY }\end{array}$

$\begin{array}{lll}3.3 & \text { Split SUSY } & 22\end{array}$

4 Mini-split with anomaly mediation $\quad 24$

$\begin{array}{lll}4.1 & \text { Phenomenology of mini-split with anomaly mediation } & 27\end{array}$

5 Conclusions $\quad 31$

$\begin{array}{ll}\text { A Loop functions } & 32\end{array}$

\section{Introduction}

The negative results of the searches for new physics at the LHC have cast some doubts on the existence of low-energy supersymmetry (SUSY) and, more generally, on the validity of the naturalness principle for the Fermi scale. However, supersymmetry finds other justifications beyond naturalness: as a dark matter (DM) candidate, as an element for gauge coupling unification, as an ingredient for stabilizing the potential from unwanted vacua at large Higgs field value, or as an ingredient of superstring theory. This has motivated renewed interest in "unnatural" setups, in which supersymmetry does not fully cure the Higgs naturalness problem. In this context, the Higgs mass measurement [1-3] has become a crucial (and sometimes the only) link between theory and experiment. This motivates our detailed study of the Higgs mass prediction in theories with unnatural supersymmetry. In particular, we will consider:

- Quasi-natural SUSY, in which supersymmetric particles are heavier than the weak scale, but not too far from it (say in the $1-30 \mathrm{TeV}$ range);

- High-Scale SUSY, in which all supersymmetric particles have masses around a common scale $\tilde{m}$, unrelated to the weak scale; 
- Split SUSY, in which only the scalar supersymmetric particles have masses of the order of $\tilde{m}$, while gauginos and higgsinos are lighter, possibly with masses near the weak scale;

- Mini-split with anomaly mediation, in which gauginos get mass from anomaly mediation at one loop and scalars from tree-level interactions.

Accurate codes have been developed to compute the SUSY prediction for the Higgs mass in the natural scenario where $\tilde{m} \approx M_{Z}$. When considering the unnatural scenario $\tilde{m} \gg M_{Z}$, such codes often become redundant and inaccurate: redundant because one can ignore effects suppressed by powers of $M_{Z} / \tilde{m}$; inaccurate because one needs to resum large logarithms of the ratio $\tilde{m} / M_{Z}$. The computation needs to be reorganized: the heavy particles are integrated out at the scale $\tilde{m}$, where they only induce threshold corrections (free of large logarithms) to the SUSY predictions for the couplings of the effective theory valid below $\tilde{m}$; suitable renormalization-group equations (RGEs) are used to evolve the couplings between the matching scale $\tilde{m}$ and the weak scale, where the running couplings are related to physical observables (i.e., the Higgs-boson mass, as well as the masses of fermions and of gauge bosons) via Standard Model (SM) calculations such as the one in ref. [4].

In this work we improve on the calculation of the threshold corrections at the scale $\tilde{m}$ by providing complete one-loop expressions for all the couplings relevant to the Higgs-mass calculation, as well as the dominant two-loop SUSY-QCD corrections to the quartic Higgs coupling $\lambda$.

Furthermore, we revisit the tuning condition in the case of Split SUSY, and we explore mini-split models with anomaly mediation, studying new possibilities for the LSP, which open new options for the DM candidate.

\section{Threshold corrections from heavy superparticles}

In this section we summarize the matching conditions for the couplings of the effective lagrangian in scenarios where some (if not all) of the supersymmetric particles are integrated out at the scale $\tilde{m}$. We work under the "unnatural" assumption $\tilde{m} \gg M_{Z}$, which induces significant simplifications with respect to the general expressions that hold in the natural scenario where $\tilde{m} \approx M_{Z}$. We complete and correct the results already presented in [5, 6].

\subsection{Lagrangian and tree-level matching}

We consider scenarios in which all of the sfermions, as well as a heavy Higgs $\operatorname{doublet} A$, are integrated out at the scale $\tilde{m}$. The surviving (and SM-like) Higgs doublet $H$ is a combination of the two doublets $H_{u}$ and $H_{d}$ of the underlying supersymmetric theory:

$$
\left(\begin{array}{l}
H \\
A
\end{array}\right)=\left(\begin{array}{rr}
\cos \beta & \sin \beta \\
-\sin \beta & \cos \beta
\end{array}\right)\left(\begin{array}{r}
-\epsilon H_{d}^{*} \\
H_{u}
\end{array}\right),
$$

where $\epsilon$ is the antisymmetric tensor with $\epsilon_{12}=1$. The mass parameter $m_{A}^{2}$ for the heavy doublet is of the order of $\tilde{m}^{2}$, whereas the mass parameter $m_{H}^{2}$ for the light doublet is 
negative and of the order of the weak scale. The potential for the doublet $H$ below the scale $\tilde{m}$ is given by the Standard Model expression

$$
V(H)=\frac{\lambda}{2}\left(H^{\dagger} H-v^{2}\right)^{2},
$$

where $v \approx 174 \mathrm{GeV}$. The tree-level mass of the physical Higgs scalar $h$ is $M_{h}^{2}=2 \lambda v^{2}$. The tree-level matching with the full supersymmetric theory at the scale $\tilde{m}$ determines the boundary condition for the quartic coupling

$$
\lambda(\tilde{m})=\frac{1}{4}\left[g_{2}^{2}(\tilde{m})+\frac{3}{5} g_{1}^{2}(\tilde{m})\right] \cos ^{2} 2 \beta,
$$

where $g_{1}$ and $g_{2}$ are the weak gauge coupling constants, assuming the SU(5) normalization for the hypercharge. Furthermore, the tree-level matching condition for the top Yukawa coupling is $g_{t}(\tilde{m})=y_{t}(\tilde{m}) \sin \beta$, where $y_{t}$ denotes the coupling of the MSSM while $g_{t}$ denotes the coupling of the low-energy effective theory.

We give expressions that can also be applied to the Split-SUSY scenario, where the fermionic superparticles are assumed to be lighter than the scalars. In such a case the effective lagrangian below the scale $\tilde{m}$ includes mass terms for the gauginos and the higgsinos, as well as Higgs-higgsino-gaugino Yukawa interactions:

$$
\begin{aligned}
\mathcal{L}^{\text {split } \supset} & -\frac{M_{3}}{2} \tilde{g}^{A} \tilde{g}^{A}-\frac{M_{2}}{2} \tilde{W}^{a} \tilde{W}^{a}-\frac{M_{1}}{2} \tilde{B} \tilde{B}-\mu \tilde{H}_{u}^{T} \epsilon \tilde{H}_{d}+ \\
& -\frac{H^{\dagger}}{\sqrt{2}}\left(\tilde{g}_{2 \mathrm{u}} \sigma^{a} \tilde{W}^{a}+\tilde{g}_{1 \mathrm{u}} \tilde{B}\right) \tilde{H}_{u}-\frac{H^{T} \epsilon}{\sqrt{2}}\left(-\tilde{g}_{2 \mathrm{~d}} \sigma^{a} \tilde{W}^{a}+\tilde{g}_{1 \mathrm{~d}} \tilde{B}\right) \tilde{H}_{d}+\text { h.c. },
\end{aligned}
$$

where gauginos and higgsinos are two-component spinors and $\sigma^{a}$ are the Pauli matrices. We consider for simplicity the case of real gaugino and higgsino mass parameters. The tree-level matching conditions for the Split-SUSY couplings at the scale $\tilde{m}$ are:

$$
\begin{aligned}
& \tilde{g}_{2 \mathrm{u}}(\tilde{m})=g_{2}(\tilde{m}) \sin \beta, \quad \tilde{g}_{1 \mathrm{u}}(\tilde{m})=\sqrt{3 / 5} g_{1}(\tilde{m}) \sin \beta, \\
& \tilde{g}_{2 \mathrm{~d}}(\tilde{m})=g_{2}(\tilde{m}) \cos \beta, \quad \tilde{g}_{1 \mathrm{~d}}(\tilde{m})=\sqrt{3 / 5} g_{1}(\tilde{m}) \cos \beta \text {. }
\end{aligned}
$$

Our results for the one-loop matching conditions should be used as follows:

- In the High-Scale SUSY scenario, the MSSM is directly matched onto the SM at the scale $\tilde{m}$, such that the couplings $\tilde{g}_{1 \mathrm{~d}}, \tilde{g}_{1 \mathrm{u}}, \tilde{g}_{2 \mathrm{~d}}, \tilde{g}_{2 \mathrm{u}}$ and $\lambda$ appearing in all oneloop threshold corrections can be replaced by their tree-level values of eq. (2.5) and eq. (2.3).

- In the Split-SUSY scenario, two different matchings must be applied:

$$
\begin{aligned}
& \mathrm{SM} \text { in } \overline{\mathrm{MS}} \\
& g_{1,2,3}, g_{t}, \lambda
\end{aligned} \longleftrightarrow \begin{gathered}
\text { Split-SUSY in } \overline{\mathrm{MS}} \\
g_{1,2,3}, g_{t}, \lambda, \tilde{g}_{1 \mathrm{~d}}, \tilde{g}_{1 \mathrm{u}}, \tilde{g}_{2 \mathrm{~d}}, \tilde{g}_{2 \mathrm{u}}
\end{gathered} \stackrel{\tilde{m}}{\longleftrightarrow} \begin{gathered}
\text { MSSM in } \overline{\mathrm{DR}} \\
g_{1,2,3}, y_{t}
\end{gathered}
$$

The intermediate theory contains higgsinos and gauginos. Thereby, their contributions must be removed from the matching conditions at $\tilde{m}$, and included at the lower energy scale at which Split SUSY is matched onto the SM. 


\subsection{One-loop matching}

To extend our analysis of heavy-SUSY scenarios beyond the leading order, we need to include in the matching conditions for the couplings the threshold corrections arising when the heavy particles are integrated out of the effective low-energy lagrangian. A one-loop computation of the matching conditions also requires that we specify a renormalization scheme for the parameters entering the tree-level part, and include appropriate counterterm contributions in the one-loop part.

In the full supersymmetric theory above the matching scale $\tilde{m}$, eqs. (2.3) and (2.5) are valid beyond tree level only if the parameters are renormalized in a SUSY-preserving scheme such as $\overline{\mathrm{DR}}$. However, to allow for the direct implementation of existing SM results in our calculations, we express all the couplings of the low-energy lagrangian, including the weak gauge couplings entering the right-hand side of eqs. (2.3) and (2.5), as running parameters renormalized in the $\overline{\mathrm{MS}}$ scheme. Since this scheme breaks supersymmetry, the conditions relating the gaugino and four-scalar couplings to the gauge couplings are not preserved beyond tree level even in the full supersymmetric theory [7]. In the $\overline{\mathrm{MS}}$ scheme the one-loop matching conditions of the gaugino and Higgs-quartic couplings must therefore be modified as described in [5]. In addition, we choose to express the right-hand-side of eqs. (2.3) and (2.5) in terms of the weak gauge couplings of the low-energy theory, as opposed to the couplings of the full supersymmetric theory. This induces additional one-loop shifts in the matching conditions in case the heavy-particle masses are not all equal to $\tilde{m}$.

Renormalization of $\tan \boldsymbol{\beta}$. The renormalization of the angle $\beta$ entering eqs. (2.3) and (2.5) requires a special discussion. In contrast to what happens in the MSSM, in the scenarios considered here it is not useful to relate $\beta$ to the vacuum expectation values of the Higgs doublets $H_{u}$ and $H_{d}$. Instead, $\beta$ should be interpreted just as a fine-tuned mixing angle that rotates the two original doublets into a light doublet $H$ and a massive doublet $A$. In a generic system of two scalars that mix with each other, the divergent part of the counterterm for the mixing angle $\theta$ is fixed by the requirement that it cancel the divergence of the antisymmetric part of the wave-function renormalization (WFR) matrix $[8,9]$

$$
\delta \theta^{\operatorname{div}}=\frac{1}{2} \frac{\Pi_{12}^{\mathrm{div}}\left(m_{1}^{2}\right)+\Pi_{12}^{\mathrm{div}}\left(m_{2}^{2}\right)}{m_{1}^{2}-m_{2}^{2}}
$$

where $\Pi_{12}^{\text {div }}\left(p^{2}\right)$ denotes the divergent part of the self-energy that mixes the two mass eigenstates characterized by mass eigenvalues $m_{1,2}^{2}$. The finite part of the counterterm defines the renormalization scheme for the mixing angle, and different choices have been discussed in the literature. For example, in refs. $[8,9]$ the finite part of the counterterm has the same form as the divergent part in eq. (2.6), while in ref. [10] the external momentum in the finite part of $\Pi_{12}\left(p^{2}\right)$ is set to the special value $\left(m_{1}^{2}+m_{2}^{2}\right) / 2$. In both cases, the renormalized mixing angle $\theta$ is scale-independent.

In our calculation we define the divergent part of the counterterm $\delta \beta$ according to eq. (2.6), but we choose instead to define the finite part in such a way that it removes entirely the contributions of the off-diagonal WFR of the Higgs doublets from the matching 
conditions for the effective couplings:

$$
\delta \beta^{\text {fin }}=\frac{\Pi_{H A}^{\mathrm{fin}}\left(m_{H}^{2}\right)}{m_{H}^{2}-m_{A}^{2}} .
$$

Loosely speaking, this defines the renormalized $\beta$ as the angle that diagonalizes the radiatively corrected Higgs mass matrix at an external momentum $p^{2}$ set equal to the light-Higgs mass parameter $m_{H}^{2}$ (in fact, the latter can be considered zero in comparison to $m_{A}^{2}$ ).

The definition in eq. (2.7) has the advantage of simplifying the threshold corrections to the matching conditions, but it leads to a scale-dependent mixing angle, which at one loop is subject to the same RGE as the usual parameter $\beta$ of the MSSM. However, it must be recalled that the angle $\beta$ is not a parameter of the low-energy lagrangian, and it enters only the matching conditions for the couplings at the scale $\tilde{m}$. Therefore, different choices of renormalization scheme can be simply compensated for by a shift in the (arbitrary) input value of $\beta$.

Threshold corrections to the quartic Higgs coupling. In the High-Scale SUSY setup where we integrate out all SUSY particles at the scale $\tilde{m}$, the loop-corrected boundary condition for the Higgs quartic coupling takes the form

$\lambda(\tilde{m})=\frac{1}{4}\left[g_{2}^{2}(\tilde{m})+\frac{3}{5} g_{1}^{2}(\tilde{m})\right] \cos ^{2} 2 \beta+\Delta \lambda^{1 \ell, \mathrm{reg}}+\Delta \lambda^{1 \ell, \phi}+\Delta \lambda^{1 \ell, \chi^{1}}+\Delta \lambda^{1 \ell, \chi^{2}}+\Delta \lambda^{2 \ell}$,

where we denote by $g_{i}$ the $\overline{\mathrm{MS}}$-renormalized gauge couplings of the effective theory valid below the scale $\tilde{m}$, and $\Delta \lambda^{1 \ell \text {, reg }}$ accounts for the conversion from the $\overline{\mathrm{DR}}$ to the $\overline{\mathrm{MS}}$ scheme, which modifies the tree-level relation of eq. (2.3) even in the supersymmetric limit:

$$
(4 \pi)^{2} \Delta \lambda^{1 \ell, \text { reg }}=-\frac{9}{100} g_{1}^{4}-\frac{3}{10} g_{1}^{2} g_{2}^{2}-\left(\frac{3}{4}-\frac{\cos ^{2} 2 \beta}{6}\right) g_{2}^{4} .
$$

Concerning the other terms in eq. (2.8), $\Delta \lambda^{1 \ell, \phi}$ is the one-loop threshold correction arising when we integrate out the heavy scalars; $\Delta \lambda^{1 \ell, \chi^{1}}$ and $\Delta \lambda^{1 \ell, \chi^{2}}$ are corrections arising when we integrate out the higgsinos and the electroweak (EW) gauginos; finally, $\Delta \lambda^{2 \ell}$ contains the dominant two-loop correction from diagrams involving stop squarks, which will be described in the next subsection.

Neglecting all Yukawa couplings except the top coupling $g_{t}$, the one-loop scalar contribution to the threshold correction to $\lambda(\tilde{m})$ is ${ }^{1}$

$$
\begin{aligned}
(4 \pi)^{2} \Delta \lambda^{1 \ell, \phi}= & 3 g_{t}^{2}\left[g_{t}^{2}+\frac{1}{2}\left(g_{2}^{2}-\frac{g_{1}^{2}}{5}\right) \cos 2 \beta\right] \ln \frac{m_{Q_{3}}^{2}}{\tilde{m}^{2}}+3 g_{t}^{2}\left[g_{t}^{2}+\frac{2}{5} g_{1}^{2} \cos 2 \beta\right] \ln \frac{m_{U_{3}}^{2}}{\tilde{m}^{2}} \\
& +\frac{\cos ^{2} 2 \beta}{300} \sum_{i=1}^{3}\left[3\left(g_{1}^{4}+25 g_{2}^{4}\right) \ln \frac{m_{Q_{i}}^{2}}{\tilde{m}^{2}}+24 g_{1}^{4} \ln \frac{m_{U_{i}}^{2}}{\tilde{m}^{2}}+6 g_{1}^{4} \ln \frac{m_{D_{i}}^{2}}{\tilde{m}^{2}}\right.
\end{aligned}
$$

\footnotetext{
${ }^{1}$ As will be explained in section 2.3, consistency with our calculation of the dominant two-loop correction $\Delta \lambda^{2 \ell}$ requires that the terms of $\mathcal{O}\left(g_{t}^{4}\right)$ in eq. (2.10) be expressed in terms of the $\overline{\mathrm{MS}}$-renormalized top Yukawa coupling of the low-energy theory and of the $\overline{\mathrm{DR}}$-renormalized stop masses and mixing.
} 


$$
\begin{gathered}
\left.+\left(9 g_{1}^{4}+25 g_{2}^{4}\right) \ln \frac{m_{L_{i}}^{2}}{\tilde{m}^{2}}+18 g_{1}^{4} \ln \frac{m_{E_{i}}^{2}}{\tilde{m}^{2}}\right] \\
+\frac{1}{4800}\left[261 g_{1}^{4}+630 g_{1}^{2} g_{2}^{2}+1325 g_{2}^{4}-4 \cos 4 \beta\left(9 g_{1}^{4}+90 g_{1}^{2} g_{2}^{2}+175 g_{2}^{4}\right)\right. \\
\left.-9 \cos 8 \beta\left(3 g_{1}^{2}+5 g_{2}^{2}\right)^{2}\right] \ln \frac{m_{A}^{2}}{\tilde{m}^{2}}-\frac{3}{16}\left(\frac{3}{5} g_{1}^{2}+g_{2}^{2}\right)^{2} \sin ^{2} 4 \beta \\
+6 g_{t}^{4} \widetilde{X}_{t}\left[\widetilde{F}_{1}\left(x_{Q U}\right)-\frac{\widetilde{X}_{t}}{12} \widetilde{F}_{2}\left(x_{Q U}\right)\right]+\frac{3}{4} g_{t}^{2} \widetilde{X}_{t} \cos 2 \beta\left[\frac{3}{5} g_{1}^{2} \widetilde{F}_{3}\left(x_{Q U}\right)+g_{2}^{2} \widetilde{F}_{4}\left(x_{Q U}\right)\right] \\
-\frac{1}{4} g_{t}^{2} \widetilde{X}_{t} \cos ^{2} 2 \beta\left(\frac{3}{5} g_{1}^{2}+g_{2}^{2}\right) \widetilde{F}_{5}\left(x_{Q U}\right) .
\end{gathered}
$$

Here: $m_{L_{i}}, m_{E_{i}}, m_{Q_{i}}, m_{U_{i}}$ and $m_{D_{i}}$ are the soft SUSY-breaking masses for the sfermions of the $i$-th generation; $\tilde{X}_{t} \equiv X_{t}^{2} /\left(m_{Q_{3}} m_{U_{3}}\right)$, where $X_{t}=A_{t}-\mu \cot \beta$ and $A_{t}$ is the soft SUSY-breaking Higgs-stop coupling; $x_{Q U} \equiv m_{Q_{3}} / m_{U_{3}}$; the loop functions $\tilde{F}_{i}$ are defined in appendix A, eq. (A.1a).

The first, second and third lines of eq. (2.10) contain threshold corrections arising when the squarks and sleptons are integrated out of the theory (including the contributions due to the redefinition of the gauge couplings); the fourth and fifth lines contain the corresponding contribution of the heavy Higgs doublet; the last two lines contain the corrections controlled by $\widetilde{X}_{t} \cdot{ }^{2}$

Finally, we give the one-loop higgsino-gaugino contributions to the matching condition for $\lambda(\tilde{m})$. The first one, containing the proper threshold corrections to the quartic coupling, was given in ref. [6] in terms of the effective couplings of Split SUSY:

$$
\begin{aligned}
(4 \pi)^{2} \Delta \lambda^{1 \ell, \chi^{1}=} & \frac{1}{2} \tilde{\beta}_{\lambda} \ln \frac{\mu^{2}}{\tilde{m}^{2}}+\left[-\frac{7}{12} f_{1}\left(r_{1}\right)\left(\tilde{g}_{1 \mathrm{~d}}^{4}+\tilde{g}_{1 \mathrm{u}}^{4}\right)-\frac{9}{4} f_{2}\left(r_{2}\right)\left(\tilde{g}_{2 \mathrm{~d}}^{4}+\tilde{g}_{2 \mathrm{u}}^{4}\right)\right. \\
& -\frac{3}{2} f_{3}\left(r_{1}\right) \tilde{g}_{1 \mathrm{~d}}^{2} \tilde{g}_{1 \mathrm{u}}^{2}-\frac{7}{2} f_{4}\left(r_{2}\right) \tilde{g}_{2 \mathrm{~d}}^{2} \tilde{g}_{2 \mathrm{u}}^{2}-\frac{8}{3} f_{5}\left(r_{1}, r_{2}\right) \tilde{g}_{1 \mathrm{~d}} \tilde{g}_{1 \mathrm{u}} \tilde{g}_{2 \mathrm{~d}} \tilde{g}_{2 \mathrm{u}} \\
& -\frac{7}{6} f_{6}\left(r_{1}, r_{2}\right)\left(\tilde{g}_{1 \mathrm{~d}}^{2} \tilde{g}_{2 \mathrm{~d}}^{2}+\tilde{g}_{1 \mathrm{u}}^{2} \tilde{g}_{2 \mathrm{u}}^{2}\right)-\frac{1}{6} f_{7}\left(r_{1}, r_{2}\right)\left(\tilde{g}_{1 \mathrm{~d}}^{2} \tilde{g}_{2 \mathrm{u}}^{2}+\tilde{g}_{1 \mathrm{u}}^{2} \tilde{g}_{2 \mathrm{~d}}^{2}\right) \\
& -\frac{4}{3} f_{8}\left(r_{1}, r_{2}\right)\left(\tilde{g}_{1 \mathrm{~d}} \tilde{g}_{2 \mathrm{u}}+\tilde{g}_{1 \mathrm{u}} \tilde{g}_{2 \mathrm{~d}}\right)\left(\tilde{g}_{1 \mathrm{~d}} \tilde{g}_{2 \mathrm{~d}}+\tilde{g}_{1 \mathrm{u}} \tilde{g}_{2 \mathrm{u}}\right) \\
& +\frac{2}{3} f\left(r_{1}\right) \tilde{g}_{1 \mathrm{~d}} \tilde{g}_{1 \mathrm{u}}\left[\lambda-2\left(\tilde{g}_{1 \mathrm{~d}}^{2}+\tilde{g}_{1 \mathrm{u}}^{2}\right)\right]+2 f\left(r_{2}\right) \tilde{g}_{2 \mathrm{~d}} \tilde{g}_{2 \mathrm{u}}\left[\lambda-2\left(\tilde{g}_{2 \mathrm{~d}}^{2}+\tilde{g}_{2 \mathrm{u}}^{2}\right)\right] \\
& \left.+\frac{1}{3} g\left(r_{1}\right) \lambda\left(\tilde{g}_{1 \mathrm{~d}}^{2}+\tilde{g}_{1 \mathrm{u}}^{2}\right)+g\left(r_{2}\right) \lambda\left(\tilde{g}_{2 \mathrm{~d}}^{2}+\tilde{g}_{2 \mathrm{u}}^{2}\right)\right]
\end{aligned}
$$

\footnotetext{
${ }^{2}$ Note that the result in eq. (2.10) corrects both eq. (2.6) of ref. [5] and eq. (7) of ref. [6]. In the former, a common mass $M_{S}$ was assumed for all of the heavy scalars, therefore most of the terms appearing in our eq. (2.10) vanish. However, a factor $-\cos 2 \beta$ was omitted in the contribution proportional to $h_{t}^{2}\left(g^{2}+g^{\prime 2}\right)$, and the non-vanishing terms in the fifth and in the last lines of our eq. (2.10) were missed. Concerning eq. (7) of ref. [6], the heavy-Higgs contribution — see the fourth and fifth lines of our eq. (2.10) — was incorrect, and the term in the last line, arising from the diagonal WFR of the external legs, was missed. Further discrepancies between our eq. (2.10) and eq. (7) of ref. [6] are due to the fact that the latter was computed under the assumption that the tree-level part of the matching condition is expressed in terms of the $\overline{\mathrm{MS}}$-renormalized gauge couplings of the MSSM, as opposed to those of the low-energy effective theory.
} 
where $r_{i} \equiv M_{i} / \mu$, and

$$
\begin{aligned}
\tilde{\beta}_{\lambda}= & 2 \lambda\left(\tilde{g}_{1 \mathrm{~d}}^{2}+\tilde{g}_{1 \mathrm{u}}^{2}+3 \tilde{g}_{2 \mathrm{~d}}^{2}+3 \tilde{g}_{2 \mathrm{u}}^{2}\right)-\tilde{g}_{1 \mathrm{~d}}^{4}-\tilde{g}_{1 \mathrm{u}}^{4}-5 \tilde{g}_{2 \mathrm{~d}}^{4}-5 \tilde{g}_{2 \mathrm{u}}^{4} \\
& -4 \tilde{g}_{1 \mathrm{~d}} \tilde{g}_{1 \mathrm{u}} \tilde{g}_{2 \mathrm{~d}} \tilde{g}_{2 \mathrm{u}}-2\left(\tilde{g}_{1 \mathrm{~d}}^{2}+\tilde{g}_{2 \mathrm{u}}^{2}\right)\left(\tilde{g}_{1 \mathrm{u}}^{2}+\tilde{g}_{2 \mathrm{~d}}^{2}\right)
\end{aligned}
$$

is the Split-SUSY contribution to the one-loop beta function of $\lambda$. The functions $f_{i}, f$ and $g$ are defined in appendix A, eq. (A.3a). In the case of High-Scale SUSY, the quartic coupling $\lambda$ and the effective higgs-higgsino-gaugino couplings entering eq. (2.11) must be expressed in terms of the gauge couplings and of the angle $\beta$ by means of eqs. (2.3) and (2.5).

The second higgsino-gaugino contribution to $\lambda(\tilde{m})$,

$$
(4 \pi)^{2} \Delta \lambda^{1 \ell, \chi^{2}}=-\frac{1}{6} \cos ^{2} 2 \beta\left[2 g_{2}^{4} \ln \frac{M_{2}^{2}}{\tilde{m}^{2}}+\left(\frac{9}{25} g_{1}^{4}+g_{2}^{4}\right) \ln \frac{\mu^{2}}{\tilde{m}^{2}}\right]
$$

arises from the fact that in High-Scale SUSY the tree-level part of the matching condition for $\lambda$ in eq. (2.8) is expressed in terms of the gauge couplings of the SM. ${ }^{3}$

In the Split-SUSY setup, the higgsino-gaugino contributions are removed from the matching condition for $\lambda(\tilde{m})$, eq. (2.8), and the tree-level part of the matching condition is expressed in terms of the gauge couplings of Split SUSY. However, $\Delta \lambda^{1 \ell, \chi^{1}}$ reappears as a threshold correction at the lower scale $m_{\text {split }}$ where the Split-SUSY lagrangian is matched to the SM lagrangian:

$$
\lambda^{\mathrm{SM}}\left(m_{\text {split }}\right)=\lambda^{\text {split }}\left(m_{\text {split }}\right)+\Delta \lambda^{1 \ell, \chi^{1}} .
$$

We remark that this procedure neglects effects suppressed by inverse powers of the superparticle masses, and is therefore accurate only if there is some hierarchy between the masses of higgsinos and gauginos and the weak scale. Full one-loop results for the charginoneutralino contributions to the Higgs mass in Split SUSY were provided in refs. [5, 11].

We also recall that, in Split SUSY, the soft SUSY-breaking parameter $A_{t}$ is suppressed by the same symmetry that keeps $\mu$ and the gaugino masses smaller than the scalar masses. Therefore, the terms proportional to $\widetilde{X}_{t}$ in the last two lines of eq. (2.10) become negligible.

Threshold corrections to the Split-SUSY couplings. In the Split-SUSY scenario one also needs to generalize the tree-level expressions of eq. (2.5) for the Higgs-higgsinogaugino couplings at the scale $\tilde{m}$ adding the one-loop threshold corrections. We find:

$$
\begin{aligned}
\frac{\tilde{g}_{2 \mathrm{u}}}{g_{2} \sin \beta}=1 & +\frac{1}{(4 \pi)^{2}}\left\{-g_{2}^{2}\left(\frac{2}{3}+\frac{11}{16} \cos ^{2} \beta\right)+\frac{3 g_{1}^{2}}{80}\left(-2+7 \cos ^{2} \beta\right)+\frac{9 g_{t}^{2}}{4 \sin ^{2} \beta}\right. \\
& +\frac{20 g_{2}^{2}+3\left(-9 g_{1}^{2}+35 g_{2}^{2}\right) \cos ^{2} \beta}{120} \ln \frac{m_{A}^{2}}{\tilde{m}^{2}}+\frac{g_{2}^{2}}{6} \sum_{i=1}^{3} \ln \frac{m_{L_{i}}^{2}}{\tilde{m}^{2}} \\
& \left.+\frac{g_{2}^{2}}{2} \sum_{i=1}^{3} \ln \frac{m_{Q_{i}}^{2}}{\tilde{m}^{2}}-\frac{3}{4} \frac{g_{t}^{2}}{\sin ^{2} \beta}\left[3 \ln \frac{m_{Q_{3}}^{2}}{\tilde{m}^{2}}-\ln \frac{m_{U_{3}}^{2}}{\tilde{m}^{2}}\right]\right\},
\end{aligned}
$$

\footnotetext{
${ }^{3}$ This contribution is not included in eq. (7) of ref. [6], due to the different definition adopted in that paper for the gauge couplings entering the tree-level matching condition for $\lambda$.
} 


$$
\begin{aligned}
\frac{\tilde{g}_{2 \mathrm{~d}}}{g_{2} \cos \beta}=1 & +\frac{1}{(4 \pi)^{2}}\left\{-g_{2}^{2}\left(\frac{2}{3}+\frac{11}{16} \sin ^{2} \beta\right)+\frac{3 g_{1}^{2}}{80}\left(-2+7 \sin ^{2} \beta\right)+\frac{g_{2}^{2}}{2} \sum_{i=1}^{3} \ln \frac{m_{Q_{i}}^{2}}{\tilde{m}^{2}}\right. \\
& \left.+\frac{20 g_{2}^{2}+3\left(-9 g_{1}^{2}+35 g_{2}^{2}\right) \sin ^{2} \beta}{120} \ln \frac{m_{A}^{2}}{\tilde{m}^{2}}+\frac{g_{2}^{2}}{6} \sum_{i=1}^{3} \ln \frac{m_{L_{i}}^{2}}{\tilde{m}^{2}}\right\}, \quad(2.16) \\
\frac{\tilde{g}_{1 \mathrm{u}}}{\sqrt{3 / 5} g_{1} \sin \beta}=1 & +\frac{1}{(4 \pi)^{2}}\left\{\frac{3 g_{2}^{2}}{16}\left(-2+7 \cos ^{2} \beta\right)+\frac{3 g_{1}^{2}}{80}\left(-44+7 \cos ^{2} \beta\right)+\frac{9 g_{t}^{2}}{4 \sin ^{2} \beta}\right. \\
& +\frac{4 g_{1}^{2}-9\left(g_{1}^{2}+5 g_{2}^{2}\right) \cos ^{2} \beta}{40} \ln \frac{m_{A}^{2}}{\tilde{m}^{2}}+\frac{g_{1}^{2}}{10} \sum_{i=1}^{3}\left[\ln \frac{m_{L_{i}}^{2}}{\tilde{m}^{2}}+2 \ln \frac{m_{E_{i}}^{2}}{\tilde{m}^{2}}\right] \\
& +\frac{g_{1}^{2}}{30} \sum_{i=1}^{3}\left[\ln \frac{m_{Q_{i}}^{2}}{\tilde{m}^{2}}+8 \ln \frac{m_{U_{i}}^{2}}{\tilde{m}^{2}}+2 \ln \frac{m_{D_{i}}^{2}}{\tilde{m}^{2}}\right] \\
& \left.+\frac{g_{t}^{2}}{4 \sin ^{2} \beta}\left(7 \ln \frac{m_{Q_{3}}^{2}}{\tilde{m}^{2}}-13 \ln \frac{m_{U_{3}}^{2}}{\tilde{m}^{2}}\right)\right\}, \\
\frac{\tilde{g}_{1 \mathrm{~d}}}{\sqrt{3 / 5} g_{1} \cos \beta}= & \frac{1}{(4 \pi)^{2}}\left\{\frac{3 g_{2}^{2}}{16}\left(-2+7 \sin ^{2} \beta\right)+\frac{3 g_{1}^{2}}{80}\left(-44+7 \sin ^{2} \beta\right)\right. \\
& +\frac{4 g_{1}^{2}-9\left(g_{1}^{2}+5 g_{2}^{2}\right) \sin ^{2} \beta}{40} \ln \frac{m_{A}^{2}}{\tilde{m}^{2}}+\frac{g_{1}^{2}}{10} \sum_{i=1}^{3}\left[\ln \frac{m_{L_{i}}^{2}}{\tilde{m}^{2}}+2 \ln \frac{m_{E_{i}}^{2}}{\tilde{m}^{2}}\right] \\
& \left.+\frac{g_{1}^{2}}{30} \sum_{i=1}^{3}\left[\ln \frac{m_{Q_{i}}^{2}}{\tilde{m}^{2}}+8 \ln \frac{m_{U_{i}}^{2}}{\tilde{m}^{2}}+2 \ln \frac{m_{D_{i}}^{2}}{\tilde{m}^{2}}\right]\right\}
\end{aligned}
$$

In the equations above we assume that the tree-level part of the matching conditions is expressed in terms of the $\overline{\mathrm{MS}}$-renormalized couplings of Split SUSY, and that the angle $\beta$ is renormalized according to the prescription in eq. (2.7). Note that the non-logarithmic terms proportional to $g_{t}^{2}$ in eqs. (2.15) and (2.17) and those proportional to $g_{1}^{2}$ and $g_{2}^{2}$ in eqs. (2.17) and (2.18) differ from the corresponding terms in eq. (11) of ref. [6], which was based on the results of ref. [12]. The discrepancies can be traced back to the fact that the renormalization of the angle $\beta$ was neglected in ref. [12], and to a mistake in eqs. (B.1) and (B.3) of that paper.

Threshold corrections to the gauge couplings. Finally, we report the one-loop matching conditions between the $\overline{\mathrm{MS}}$-renormalized gauge and Yukawa couplings of the effective theory valid below the SUSY scale, $g_{1,2,3}$ and $g_{t}$, and the corresponding $\overline{\mathrm{DR}}$ renormalized couplings of the MSSM, which we denote by $\hat{g}_{1,2,3}$ and $\hat{y}_{t}=\hat{g}_{t} / \sin \beta .{ }^{4}$ Such corrections are not needed for studying the Higgs mass prediction, but they are needed for studying issues that involve the running couplings at large energy - for example gauge coupling unification or the evolution of the soft parameters above the matching scale $\tilde{m}$.

In the High-Scale SUSY scenario, where gauginos and higgsinos are integrated out at the scale $\tilde{m}$ together with the heavy scalars, the threshold corrections to the gauge

\footnotetext{
${ }^{4}$ We can neglect the bottom Yukawa coupling because the observed value of the Higgs mass suggests a small $\tan \beta$ if the SUSY scale is large, so that $\hat{y}_{b}=\hat{g}_{b} / \cos \beta$ cannot be enhanced by a large $\tan \beta$.
} 
couplings are well known:

$$
\begin{aligned}
& \hat{g}_{1}(\tilde{m})=g_{1}(\tilde{m})+\frac{3}{5} \frac{g_{1}^{3}}{16 \pi^{2}}[-\frac{1}{3} \ln \frac{\mu^{2}}{\tilde{m}^{2}}-\frac{1}{12} \ln \frac{m_{A}^{2}}{\tilde{m}^{2}}-\frac{1}{12} \sum_{i=1}^{3}\left(\ln \frac{m_{L_{i}}^{2}}{\tilde{m}^{2}}+2 \ln \frac{m_{E_{i}}^{2}}{\tilde{m}^{2}}\right) \\
&\left.-\frac{1}{36} \sum_{i=1}^{3}\left(\ln \frac{m_{Q_{i}}^{2}}{\tilde{m}^{2}}+8 \ln \frac{m_{U_{i}}^{2}}{\tilde{m}^{2}}+2 \ln \frac{m_{D_{i}}^{2}}{\tilde{m}^{2}}\right)\right], \\
& \hat{g}_{2}(\tilde{m})=g_{2}(\tilde{m})+\frac{g_{2}^{3}}{16 \pi^{2}}\left[\frac{1}{3}-\frac{2}{3} \ln \frac{M_{2}^{2}}{\tilde{m}^{2}}-\frac{1}{3} \ln \frac{\mu^{2}}{\tilde{m}^{2}}-\frac{1}{12} \ln \frac{m_{A}^{2}}{\tilde{m}^{2}}\right. \\
&\left.\quad-\frac{1}{12} \sum_{i=1}^{3}\left(3 \ln \frac{m_{Q_{i}}^{2}}{\tilde{m}^{2}}+\ln \frac{m_{L_{i}}^{2}}{\tilde{m}^{2}}\right)\right], \\
& \hat{g}_{3}(\tilde{m})=g_{3}(\tilde{m})+\frac{g_{3}^{3}}{16 \pi^{2}}\left[\frac{1}{2}-\ln \frac{M_{3}^{2}}{\tilde{m}^{2}}-\frac{1}{12} \sum_{i=1}^{3}\left(2 \ln \frac{m_{Q_{i}}^{2}}{\tilde{m}^{2}}+\ln \frac{m_{U_{i}}^{2}}{\tilde{m}^{2}}+\ln \frac{m_{D_{i}}^{2}}{\tilde{m}^{2}}\right)\right] .
\end{aligned}
$$

The non-logarithmic terms in eqs. (2.20) and (2.21) account for the $\overline{\mathrm{MS}}-\overline{\mathrm{DR}}$ conversion of $g_{2}$ and $g_{3}$.

In the Split-SUSY scenario the logarithmic terms involving the higgsino and gaugino masses $\mu, M_{2}$ and $M_{3}$ must be removed from eqs. (2.19)-(2.21), and they reappear at the Split-SUSY scale as threshold corrections between the gauge couplings of Split SUSY and the corresponding couplings of the SM (both defined in the $\overline{\mathrm{MS}}$ scheme).

Threshold corrections to the top Yukawa coupling. The one-loop relation between the $\overline{\mathrm{DR}}$-renormalized top Yukawa coupling of the MSSM and the $\overline{\mathrm{MS}}$-renormalized coupling of the effective theory valid below $\tilde{m}$ involves a contribution arising from the $\overline{\mathrm{MS}}-\overline{\mathrm{DR}}$ conversion, one arising from corrections involving the heavy scalars, and one arising from corrections involving only higgsinos and gauginos:

$$
\hat{y}_{t}(\tilde{m})=\frac{g_{t}(\tilde{m})}{\sin \beta}\left(1+\Delta g_{t}^{\mathrm{reg}}+\Delta g_{t}^{\phi}+\Delta g_{t}^{\chi}\right),
$$

where the angle $\beta$ entering the tree-level part of the relation is renormalized according to the prescription in eq. (2.7). We find:

$$
\begin{aligned}
(4 \pi)^{2} \Delta g_{t}^{\text {reg }}= & \frac{g_{1}^{2}}{120}+\frac{3 g_{2}^{2}}{8}-\frac{4 g_{3}^{2}}{3}, \\
(4 \pi)^{2} \Delta g_{t}^{\phi}=- & \frac{4}{3} g_{3}^{2}\left[\ln \frac{M_{3}^{2}}{\tilde{m}^{2}}+\widetilde{F}_{6}\left(\frac{m_{Q_{3}}}{M_{3}}\right)+\widetilde{F}_{6}\left(\frac{m_{U_{3}}}{M_{3}}\right)-\frac{X_{t}}{M_{3}} \widetilde{F}_{9}\left(\frac{m_{Q_{3}}}{M_{3}}, \frac{m_{U_{3}}}{M_{3}}\right)\right] \\
& -g_{2}^{2}\left[\frac{3}{8} \ln \frac{M_{2}^{2}}{\tilde{m}^{2}}-\frac{3}{2} \ln \frac{\mu^{2}}{\tilde{m}^{2}}+\frac{3}{4} \widetilde{F}_{6}\left(\frac{m_{Q_{3}}}{M_{2}}\right)-\frac{3}{4} \widetilde{F}_{8}\left(\frac{m_{Q_{3}}}{\mu}, \frac{M_{2}}{\mu}\right)\right. \\
& \left.-\frac{3}{4} \frac{M_{2}}{\mu} \cot \beta \widetilde{F}_{9}\left(\frac{m_{Q_{3}}}{\mu}, \frac{M_{2}}{\mu}\right)\right] \\
- & \frac{3}{5} g_{1}^{2}\left[\frac{17}{72} \ln \frac{M_{1}^{2}}{\tilde{m}^{2}}-\frac{1}{2} \ln \frac{\mu^{2}}{\tilde{m}^{2}}+\frac{1}{36} \widetilde{F}_{6}\left(\frac{m_{Q_{3}}}{M_{1}}\right)+\frac{4}{9} \widetilde{F}_{6}\left(\frac{m_{U_{3}}}{M_{1}}\right)\right. \\
& +\frac{1}{12} \widetilde{F}_{8}\left(\frac{m_{Q_{3}}}{\mu}, \frac{M_{1}}{\mu}\right)-\frac{1}{3} \widetilde{F}_{8}\left(\frac{m_{U_{3}}}{\mu}, \frac{M_{1}}{\mu}\right)-\frac{X_{t}}{9 M_{1}} \widetilde{F}_{9}\left(\frac{m_{Q_{3}}}{M_{1}}, \frac{m_{U_{3}}}{M_{1}}\right)
\end{aligned}
$$




$$
\begin{aligned}
&\left.+\frac{M_{1} \cot \beta}{12 \mu} \widetilde{F}_{9}\left(\frac{m_{Q_{3}}}{\mu}, \frac{M_{1}}{\mu}\right)-\frac{M_{1} \cot \beta}{3 \mu} \widetilde{F}_{9}\left(\frac{m_{U_{3}}}{\mu}, \frac{M_{1}}{\mu}\right)\right] \\
&-g_{t}^{2}\left[\frac{3}{4 \sin ^{2} \beta} \ln \frac{\mu^{2}}{\tilde{m}^{2}}+\frac{3}{8} \cot ^{2} \beta\left(2 \ln \frac{m_{A}^{2}}{\tilde{m}^{2}}-1\right)\right. \\
&\left.-\frac{\widetilde{X}_{t}}{4} \widetilde{F}_{5}\left(\frac{m_{Q_{3}}}{m_{U_{3}}}\right)+\frac{1}{\sin ^{2} \beta} \widetilde{F}_{6}\left(\frac{m_{Q_{3}}}{\mu}\right)+\frac{1}{2 \sin ^{2} \beta} \widetilde{F}_{6}\left(\frac{m_{U_{3}}}{\mu}\right)\right], \\
&(4 \pi)^{2} \Delta g_{t}^{\chi}=-\frac{1}{6} \tilde{g}_{1 \mathrm{u}} \tilde{g}_{1 \mathrm{~d}} f\left(\frac{M_{1}}{\mu}\right)-\frac{1}{12}\left(\tilde{g}_{1 \mathrm{u}}^{2}+\tilde{g}_{1 \mathrm{~d}}^{2}\right)\left[g\left(\frac{M_{1}}{\mu}\right)+3 \ln \frac{\mu^{2}}{\tilde{m}^{2}}\right] \\
&-\frac{1}{2} \tilde{g}_{2 \mathrm{u}} \tilde{g}_{2 \mathrm{~d}} f\left(\frac{M_{2}}{\mu}\right)-\frac{1}{4}\left(\tilde{g}_{2 \mathrm{u}}^{2}+\tilde{g}_{2 \mathrm{~d}}^{2}\right)\left[g\left(\frac{M_{2}}{\mu}\right)+3 \ln \frac{\mu^{2}}{\tilde{m}^{2}}\right],
\end{aligned}
$$

where all loop functions are defined in appendix A, eqs. (A.1a)-(A.3a).

Once again, in High-Scale SUSY the Higgs-higgsino-gaugino couplings entering eq. (2.25) must be expressed in terms of the gauge couplings and of $\beta$ by means of eq. (2.5). In Split SUSY, on the other hand, the term $\Delta g_{t}^{\chi}$ must be removed from the boundary condition at the scale $\tilde{m}$, but it enters the relation between the top Yukawa coupling of Split SUSY and the corresponding coupling of the SM at the intermediate matching scale: ${ }^{5}$

$$
g_{t}^{\mathrm{SM}}\left(m_{\mathrm{split}}\right)=g_{t}^{\mathrm{split}}\left(m_{\mathrm{split}}\right)\left(1-\Delta g_{t}^{\chi}\right) .
$$

\subsection{Two-loop SUSY-QCD correction to the quartic Higgs coupling}

To further improve the accuracy of our prediction for the Higgs mass, we compute the $\mathcal{O}\left(g_{3}^{2} g_{t}^{4}\right)$ two-loop contribution to the matching condition for the quartic coupling of the light Higgs. Since there are no WFR contributions to the matching condition at this order in the couplings, the calculation can be performed entirely in the effective-potential approach, exploiting the techniques employed in refs. $[13,14]$ for the calculation of the Higgs masses in the MSSM and in the NMSSM.

The $\mathcal{O}\left(g_{3}^{2} g_{t}^{4}\right)$ threshold correction to the light-Higgs quartic coupling $\lambda$ at the matching scale $\tilde{m}$ can be expressed as

$$
\Delta \lambda^{2 \ell}=\left.\frac{1}{2} \frac{\partial^{4} \Delta V^{2 \ell, \tilde{t}}}{\partial^{2} H^{\dagger} \partial^{2} H}\right|_{H=0}+\Delta \lambda^{2 \ell, \text { shift }},
$$

where $\Delta V^{2 \ell, \tilde{t}}$ denotes the contribution to the MSSM scalar potential from two-loop diagrams involving the strong gauge interactions of the stop squarks,

$$
\begin{aligned}
\Delta V^{2 \ell, \tilde{t}}= & \frac{g_{3}^{2}}{64 \pi^{4}}\left\{2 m_{\tilde{t}_{1}}^{2} I\left(m_{\tilde{t}_{1}}^{2}, m_{\tilde{t}_{1}}^{2}, 0\right)+2 L\left(m_{\tilde{t}_{1}}^{2}, M_{3}^{2}, m_{t}^{2}\right)-4 m_{t} M_{3} s_{2 \theta} I\left(m_{\tilde{t}_{1}}^{2}, M_{3}^{2}, m_{t}^{2}\right)\right. \\
& \left.+\left(1-\frac{s_{2 \theta}^{2}}{2}\right) J\left(m_{\tilde{t}_{1}}^{2}, m_{\tilde{t}_{1}}^{2}\right)+\frac{s_{2 \theta}^{2}}{2} J\left(m_{\tilde{t}_{1}}^{2}, m_{\tilde{t}_{2}}^{2}\right)+\left[m_{\tilde{t}_{1}} \leftrightarrow m_{\tilde{t}_{2}}, s_{2 \theta} \rightarrow !-s_{2 \theta}\right]\right\},
\end{aligned}
$$

while $\Delta \lambda^{2 \ell \text {, shift }}$ contains additional two-loop contributions that will be described below. The loop integrals $I(x, y, z), L(x, y, z)$ and $J(x, y)$ in eq. (2.28) are defined, e.g., in appendix

\footnotetext{
${ }^{5}$ Our $\Delta g_{t}^{\chi}$ corresponds to the $\tilde{\delta}_{t}$ given in eq. (24) of ref. [6].
} 
D of ref. [14], $M_{3}$ stands for the gluino mass, $m_{\tilde{t}_{1}}$ and $m_{\tilde{t}_{2}}$ are the two stop-mass eigenstates, and $s_{2 \theta} \equiv \sin 2 \theta_{\tilde{t}}$, where $\theta_{\tilde{t}}$ denotes the stop mixing angle. The latter is related to the other parameters by

$$
\sin 2 \theta_{\tilde{t}}=\frac{2 m_{t}\left(A_{t}-\mu \cot \beta\right)}{m_{\tilde{t}_{1}}^{2}-m_{\tilde{t}_{2}}^{2}} .
$$

Since we consider scenarios in which electroweak symmetry breaking (EWSB) occurs only along the direction of the light Higgs doublet $H$, the calculation of two-loop corrections to its couplings in the effective-potential approach is considerably simplified with respect to the MSSM and NMSSM cases. We can express $m_{\tilde{t}_{1}}, m_{\tilde{t}_{2}}$ and $s_{2 \theta}$ as functions of a field-dependent top mass $m_{t}=\hat{g}_{t}|H|$, where $\hat{g}_{t}=\hat{y}_{t} \sin \beta$, and re-write eq. (2.27) as

$$
\Delta \lambda^{2 \ell}=\left.\frac{\hat{g}_{t}^{4}}{2}\left(2 \mathcal{D}_{2}+4 m_{t}^{2} \mathcal{D}_{3}+m_{t}^{4} \mathcal{D}_{4}\right) \Delta V^{2 \ell, \tilde{t}}\right|_{m_{t} \rightarrow 0}+\Delta \lambda^{2 \ell, \text { shift }},
$$

where we define the operators

$$
\mathcal{D}_{i} \equiv\left(\frac{d}{d m_{t}^{2}}\right)^{i}
$$

We then exploit the following relations for the derivatives of the field-dependent parameters:

$$
\frac{d m_{\tilde{t}_{1,2}}^{2}}{d m_{t}^{2}}=1 \pm \frac{s_{2 \theta}}{2 m_{t}}\left(A_{t}-\mu \cot \beta\right), \quad \frac{d s_{2 \theta}}{d m_{t}^{2}}=\frac{s_{2 \theta}}{2 m_{t}^{2}}\left(1-s_{2 \theta}^{2}\right) .
$$

In order to obtain the limit $m_{t} \rightarrow 0$ in eq. (2.30) - of course, after taking derivatives with respect to $m_{t}^{2}$ - we use eq. (2.29) to make the dependence of $s_{2 \theta}$ on $m_{t}$ explicit, we expand the function $\Phi\left(m_{\tilde{t}_{i}}^{2}, M_{3}^{2}, m_{t}^{2}\right)$ entering the loop integrals (see appendix D of ref. [14]) in powers of $m_{t}^{2}$, and finally we identify $m_{\tilde{t}_{1}}$ and $m_{\tilde{t}_{2}}$ with the soft SUSY-breaking stop masses $m_{Q_{3}}$ and $m_{U_{3}}$. It turns out that the combination of derivatives of $\Delta V^{2 \ell, \tilde{t}}$ in the right-hand side of eq. (2.30) contains terms proportional to $\ln \left(m_{t}^{2} / \tilde{m}^{2}\right)$, which diverge for $m_{t} \rightarrow 0$. However, we must take into account that above the matching scale $\tilde{m}$ the one-loop contribution to $\lambda$ from the box diagram with a top quark,

$$
\delta \lambda^{g_{t}^{4}, t}=-\frac{3 \hat{g}_{t}^{4}}{16 \pi^{2}}\left(2 \ln \frac{m_{t}^{2}}{\tilde{m}^{2}}+3\right)
$$

is expressed in terms of the top Yukawa coupling of the MSSM, $\hat{g}_{t}$, whereas below $\tilde{m}$ the same contribution is expressed in terms of the corresponding coupling of the low-energy theory, $g_{t}$. Being present both above and below the matching scale, $\delta \lambda^{g_{t}^{4}, t}$ does not affect the one-loop threshold correction to $\lambda$. However, to compute the matching condition at the two-loop level we must re-express the MSSM coupling entering $\delta \lambda^{g_{t}^{4}, t}$ above $\tilde{m}$ (including the coupling implicit in $\left.m_{t}^{2}\right)$ according to $\hat{g}_{t} \rightarrow g_{t}\left(1+\Delta g_{t}^{\phi, g_{s}^{2}}\right)$, where $\Delta g_{t}^{\phi, g_{s}^{2}}$ denotes the terms proportional to $g_{s}^{2}$ in eq. (2.24). This induces a two-loop contribution to $\Delta \lambda^{2 \ell}$ which cancels out the terms proportional to $\ln \left(m_{t}^{2} / \tilde{m}^{2}\right)$ in the derivatives of the effective potential. In addition, we re-express the MSSM coupling entering the terms proportional to $\hat{g}_{t}^{4}$ in the one-loop stop contribution to $\lambda$, see eq. (2.10), according to $\hat{g}_{t} \rightarrow g_{t}\left(1+\Delta g_{t}^{\phi, g_{s}^{2}}+\Delta g_{t}^{\text {ren, } g_{s}^{2}}\right)$. The correction $\Delta g_{t}^{\text {ren, } g_{s}^{2}}$ denotes the term proportional to $g_{s}^{2}$ in eq. (2.23), and accounts 
for the fact that we renormalize the couplings of the low-energy theory in the $\overline{\mathrm{MS}}$ scheme, while the effective-potential calculation of the two-loop contributions to $\lambda$ was performed in the $\overline{\mathrm{DR}}$ scheme. The combined effect of these shifts is the term denoted as $\Delta \lambda^{2 \ell \text {, shift }}$ in eqs. (2.27) and (2.30). Note that the $\overline{\mathrm{DR}}-\overline{\mathrm{MS}}$ redefinition of the Yukawa coupling in the one-loop top contribution $\delta \lambda^{g_{t}^{4}, t}$ has the same effect above and below the matching scale, therefore it does not contribute to $\Delta \lambda^{2 \ell, \text { shift }}$.

It is interesting to remark that the two-loop contributions arising from the operators $\mathcal{D}_{3}$ and $\mathcal{D}_{4}$ in eq. (2.30) cancel out completely against the shift induced when the corresponding contributions in the one-loop part - in practice, the non-logarithmic term in $\delta \lambda^{g_{t}^{4}, t}$, see eq. (2.33) - are expressed in terms of the top Yukawa coupling of the low-energy theory. Consequently, the final result for $\Delta \lambda^{2 \ell}$ originates only from the operator $\mathcal{D}_{2}$, and is therefore proportional to the stop contribution to the $\mathcal{O}\left(g_{3}^{2} g_{t}^{2} m_{t}^{2}\right)$ correction to the light-Higgs mass in the MSSM. This "decoupling" property of the two-loop SUSY contribution to the lightHiggs quartic coupling was also noted, in a slightly different context, in ref. [15].

In the Split-SUSY case, we can take the limit of vanishing gluino mass in the two-loop correction to the Higgs quartic coupling. We obtain ${ }^{6}$

$$
\begin{aligned}
\Delta \lambda^{2 \ell}=-\frac{g_{3}^{2} g_{t}^{4}}{32 \pi^{4}}\left\{3+4 \ln x_{Q U}+8 \ln ^{2} x_{Q U}+6 \ln ^{2} \frac{m_{Q 3}^{2}}{\tilde{m}^{2}}-4\left(1+3 \ln x_{Q U}\right) \ln \frac{m_{Q 3}^{2}}{\tilde{m}^{2}}\right. \\
+\tilde{X}_{t}\left[\frac{12 x_{Q U} \ln x_{Q U}}{x_{Q U}^{2}-1}\left(2 \ln \frac{m_{Q_{3}}^{2}}{\tilde{m}^{2}}-1\right)-\frac{16 x_{Q U}\left(x_{Q U}^{2}-2\right) \ln ^{2} x_{Q U}}{\left(x_{Q U}^{2}-1\right)^{2}}\right] \\
+\tilde{X}_{t}^{2}\left[\frac{6 x_{Q U}^{2}\left(5+x_{Q U}^{2}\right) \ln x_{Q U}}{\left(x_{Q U}^{2}-1\right)^{3}}+\frac{4 x_{Q U}^{2}\left(x_{Q U}^{4}-4 x_{Q U}^{2}-5\right) \ln ^{2} x_{Q U}}{\left(x_{Q U}^{2}-1\right)^{4}}\right. \\
\left.\left.-\frac{10 x_{Q U}^{2}}{\left(x_{Q U}^{2}-1\right)^{2}}+\frac{12 x_{Q U}^{2}}{\left(x_{Q U}^{2}-1\right)^{2}}\left(1-\frac{x_{Q U}^{2}+1}{x_{Q U}^{2}-1} \ln x_{Q U}\right) \ln \frac{m_{Q 3}^{2}}{\tilde{m}^{2}}\right]\right\},
\end{aligned}
$$

which for equal stop masses $m_{Q_{3}}=m_{U_{3}}=\tilde{m}$ reduces to

$$
\Delta \lambda^{2 \ell}=-\frac{g_{3}^{2} g_{t}^{4}}{32 \pi^{4}}\left[3-2 \widetilde{X}_{t}+\frac{\widetilde{X}_{t}^{2}}{6}\right] .
$$

In the case of High-Scale SUSY, on the other hand, we cannot consider the gluino mass much smaller than the stop masses. The formula for $\Delta \lambda^{2 \ell, \text { HSS }}$ with full dependence on $M_{3}$, $m_{Q_{3}}$ and $m_{U_{3}}$ is lengthy and not particularly illuminating, but in the limit $M_{3}=m_{Q_{3}}=$ $m_{U_{3}}=\tilde{m}$ it simplifies to

$$
\Delta \lambda^{2 \ell, \mathrm{HSS}}=\frac{g_{3}^{2} g_{t}^{4}}{96 \pi^{4}}\left[-12 \frac{X_{t}}{\tilde{m}}-6 \frac{X_{t}^{2}}{\tilde{m}^{2}}+14 \frac{X_{t}^{3}}{\tilde{m}^{3}}+\frac{1}{2} \frac{X_{t}^{4}}{\tilde{m}^{4}}-\frac{X_{t}^{5}}{\tilde{m}^{5}}\right]
$$

It is easy to check that, consistently with the "decoupling" behavior discussed above, the $\mathcal{O}\left(g_{3}^{2} g_{t}^{4}\right)$ threshold correction to the light-Higgs quartic coupling in eq. (2.36) could be recovered directly from the known results for the $\mathcal{O}\left(g_{3}^{2} g_{t}^{2} m_{t}^{2}\right)$ correction to the light-Higgs

\footnotetext{
${ }^{6}$ We henceforth drop the distinction between $\hat{g}_{t}$ and $g_{t}$ in $\Delta \lambda^{2 \ell}$, because it amounts to a higher-order effect.
} 
mass. In particular, it is sufficient to subtract the top-quark contribution given, e.g., in eq. (20) of ref. [16] from the full MSSM correction given, e.g., in eq. (21) of ref. [17] (in the latter it is also necessary to transform the $\overline{\mathrm{DR}}$ top mass of the MSSM, denoted by $m_{t}$, into the $\overline{\mathrm{MS}}$ top mass of the SM, denoted by $\bar{m}_{t}$ ).

\section{The Higgs mass and supersymmetry}

\subsection{Quasi-natural SUSY}

In "quasi-natural" supersymmetry all superparticles have masses $\tilde{m}$ in the range between a few to tens of TeV. A combination of SUSY-breaking parameters must be fine-tuned at 1 part in $\left(\tilde{m} / M_{Z}\right)^{2}$ in order to achieve the correct $Z$-boson mass. In such scenarios, a fixed-order calculation of the MSSM prediction for the Higgs mass is no longer accurate, because corrections enhanced by $\ln \left(\tilde{m} / M_{Z}\right)$ must be resummed. This can be done with the strategy of ref. [6], including now higher-order corrections:

1. We assume that physics at the weak scale is described by the SM, and extract from data the $\overline{\mathrm{MS}}$-renormalized parameters with two-loop precision in all couplings, adopting the results of $[4] .^{7}$

2. We evolve the SM parameters from the weak scale up to the SUSY scale $\tilde{m}$ using the known RGEs of the SM at three loops.

3. At $\tilde{m}$ we equate the quartic Higgs coupling $\lambda$ with its supersymmetric prediction, as computed in section 2 including all superparticle thresholds at one loop, and the QCD superparticle thresholds at two loops.

Depending on the specific analysis being performed, the third step either determines one of the SUSY parameters (e.g., $\tan \beta$ ) at the scale $\tilde{m}$ or determines the physical Higgs mass corresponding to a given set of SUSY parameters (in this case, the input Higgs mass in the first step is varied until the value of $\lambda(\tilde{m})$ obtained by RG evolution matches the SUSY prediction).

In figure 1 we consider a simplified scenario with $\tan \beta=20$ and a degenerate superparticle spectrum (i.e., all SUSY mass parameters, including $m_{A}$ and $\mu$, equal to a common mass $\tilde{m}$ ), and show the Higgs mass as a function of $\tilde{m}$. For a given value of $\tilde{m}$, we vary the stop mixing parameter $X_{t}=A_{t}-\mu \cot \beta$ to obtain the minimal (red lines) and maximal (blue lines) values of the Higgs mass. The former are obtained in the vicinity of $X_{t}=0$, and the latter in the vicinity of $X_{t}=\sqrt{6} \tilde{m}$, i.e., the value that maximizes the dominant $\mathcal{O}\left(g_{t}^{4}\right)$ threshold correction to $\lambda(\tilde{m})$ in eq. (2.10). In both the minimal- and maximal-mixing cases, the solid line includes the effect of the two-loop SUSY-QCD corrections to $\lambda(\tilde{m})$ described in section 2.3, while the dashed line does not include it. The bands around the solid lines represent the parametric uncertainty of the prediction for the Higgs mass, obtained by varying the pole top mass and the strong gauge coupling within their $1 \sigma$ experimental

\footnotetext{
${ }^{7}$ Three-loop QCD corrections to $g_{t}$ and to $\lambda$ are also partially available and confirm the estimated higher-order uncertainties.
} 


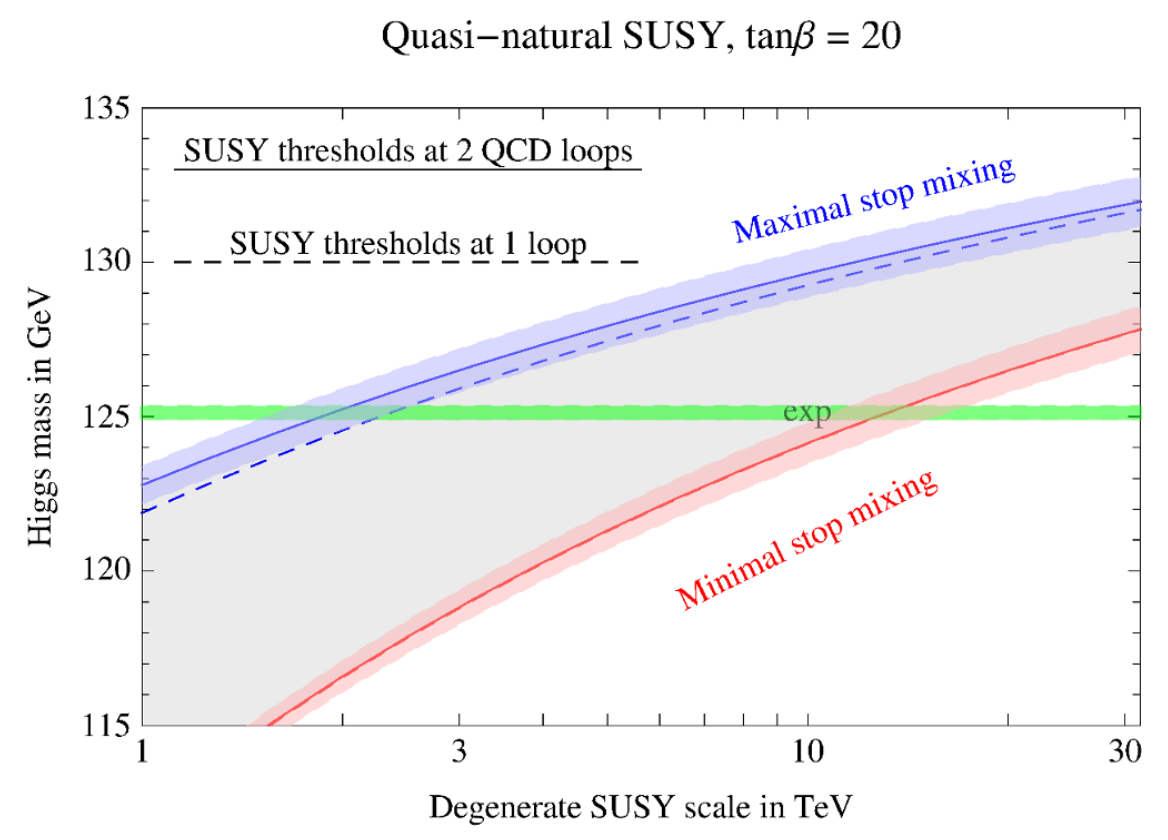

Figure 1. The Higgs mass as a function of the SUSY scale, with a degenerate spectrum of superparticles and $\tan \beta=20$. We vary the stop mixing parameter $X_{t}$ in such a way as to obtain minimal $M_{h}$ (red lines) and maximal $M_{h}$ (blue lines). The solid (dashed) lines include (neglect) the effect of the two-loop SUSY-QCD corrections to $\lambda$. The solid and dashed red lines overlap. The red and blue bands around the solid lines indicate the uncertainty associated to the measurement of the SM input parameters. The green band indicates the measured Higgs mass.

uncertainty, $M_{t}=173.34 \pm 0.76 \mathrm{GeV}$ [18] and $\alpha_{3}\left(M_{Z}\right)=0.1184 \pm 0.0007$ [19]. The green horizontal band indicates the measured value for the Higgs mass, $M_{h}^{\exp }=125.15 \pm 0.25 \mathrm{GeV}$, obtained from a naive average of the ATLAS and CMS results [1-3].

The figure suggests that, for moderately large $\tan \beta$ and degenerate SUSY masses, a value of $\tilde{m}$ around $2 \mathrm{TeV}$ is needed to predict a Higgs mass compatible with the experimental result, as long as the Higgs-stop coupling $X_{t}$ is adjusted to maximize the correction. In the case of vanishing $X_{t}$, on the other hand, SUSY masses greater than $10 \mathrm{TeV}$ are needed. In both cases, a wider range of values of $\tilde{m}$ becomes acceptable when the experimental uncertainty of $M_{t}$ and $\alpha_{3}$ is taken into account. Lowering $\tan \beta$ would reduce the tree-level part of the boundary condition for $\lambda$, requiring even larger SUSY masses.

The comparison between the solid and dashed blue lines in figure 1 shows that, for large $X_{t}$, the two-loop $\mathcal{O}\left(g_{3}^{2} g_{t}^{4}\right)$ corrections to $\lambda(\tilde{m})$ can increase the Higgs mass by up to $1 \mathrm{GeV}$ at low $\tilde{m}$, but their effect is reduced as $\tilde{m}$ gets larger (indeed, both $g_{t}$ and $g_{3}$ decrease at higher scales). On the other hand, eq. (2.36) shows that those corrections vanish for $X_{t}=0$ and degenerate SUSY masses. Consequently, the solid and dashed red lines overlap in the figure.

Comparison with other recent computations. It is useful to compare our results for the Higgs mass with those in two recent papers [20,21] where the importance of resumming 
the large logarithms in heavy-SUSY scenarios was emphasized. ${ }^{8}$ The renormalization-group (RG) calculation in ref. [20] is conceptually similar to ours, although the SM relation between the running quartic coupling and the pole Higgs mass in step 1 is computed only at one loop, and two-loop terms of $\mathcal{O}\left(g_{t}^{6}\right)$, which we neglect, are included in the SUSY correction to $\lambda(\tilde{m})$ in step 3. Ref. [21], on the other hand, combines the "diagrammatic" calculation of the MSSM Higgs masses implemented in the code FeynHiggs [23] — which includes full one-loop [24] plus dominant two-loop [13, 25-30] corrections - with a resummation of the leading and next-to-leading logarithmic terms controlled exclusively by $g_{t}$ and $g_{3}$.

We again focus on a simplified scenario with heavy and degenerate SUSY masses, $\tilde{m}=$ $10 \mathrm{TeV}$, and take $X_{t}=0$ and $\tan \beta=20$. Fixing the SM input parameters to their central values, we find $M_{h}=123.6 \mathrm{GeV}$, which should be compared to the value $M_{h}=123.2 \mathrm{GeV}$ in the upper-left plot of figure 1 in ref. [20], and to the value $M_{h}=126.5 \mathrm{GeV}$ obtained with the version of FeynHiggs described in ref. [21]. ${ }^{9}$ While the agreement between our result and the one of ref. [20] appears satisfactory in view of the small differences between the two RG calculations, the $\sim 3 \mathrm{GeV}$ discrepancy with the "hybrid" (i.e., diagrammatic+RG) calculation of ref. [21] deserves further discussion.

A decade ago, the theoretical uncertainty of partial two-loop calculations of the MSSM Higgs mass such as the one implemented in FeynHiggs was indeed estimated to be of the order of $3 \mathrm{GeV}[32,33]$. However, that estimate was developed for fixed-order calculations in what were then considered natural regions of the MSSM parameter space, and it does not necessarily apply to RG calculations in heavy-SUSY scenarios. A realistic assessment of the theoretical uncertainty of our Higgs-mass calculation should take into account three sources of uncertainty: the first are missing higher-order terms in the SM computations of steps 1 and 2, which were estimated in ref. [4] to induce an uncertainty of $\pm 0.2 \mathrm{GeV}$ in the Higgs mass. The second are missing higher-order corrections in the SUSY thresholds of step 3: by varying the matching scale by a factor of 2 around $\tilde{m}$, we estimate that these missing corrections induce an uncertainty of $\pm 0.5 \mathrm{GeV}$ in the Higgs mass. Indeed, we would not expect their effect to be much larger than the one of the known two-loop $\mathcal{O}\left(g_{3}^{2} g_{t}^{4}\right)$ corrections, which, even for large stop mixing, shift the Higgs mass by at most $0.4 \mathrm{GeV}$ in the scenario with SUSY masses all equal to $\tilde{m}=10 \mathrm{TeV}$. Finally, a third source of uncertainty are effects suppressed by powers of $v^{2} / \tilde{m}^{2}$ and by a loop factor, which arise because in steps 1 and 2 we employ the SM as an effective theory, thus neglecting heavysuperparticle effects in the determination of the running couplings, and because in step 3 we neglect the effects of EWSB when matching the MSSM couplings onto the SM ones. Of course, the relevance of $\mathcal{O}\left(v^{2} / \tilde{m}^{2}\right)$ effects decreases for increasing superparticle masses: we estimate that for $\tilde{m}=10 \mathrm{TeV}$ the uncertainty in the Higgs mass induced by those effects is already negligible. Putting all together, the theoretical uncertainty of our result for the Higgs mass in the point with $\tilde{m}=10 \mathrm{TeV}, X_{t}=0$ and $\tan \beta=20$ should not be

\footnotetext{
${ }^{8}$ An earlier study of heavy-SUSY scenarios, ref. [22], neglected the resummation of large logarithms, thus overestimating the Higgs mass by more than $10 \mathrm{GeV}$ for stop masses around $10 \mathrm{TeV}$.

${ }^{9}$ To perform the comparison, we converted the $\overline{\mathrm{DR}}$ input parameters $\tilde{m}$ and $X_{t}$ to the "on-shell" scheme adopted by FeynHiggs, using results from ref. [31]. However, in this point the effect of the conversion on the Higgs mass amounts only to a few hundred $\mathrm{MeV}$.
} 
larger than $\pm 1 \mathrm{GeV}$, which makes it incompatible with the corresponding result of ref. [21]. The observed $3-\mathrm{GeV}$ discrepancy might be explained by the fact that the resummation procedure in ref. [21] covers only a subset of the leading and next-to-leading $\ln \left(\tilde{m} / M_{t}\right)$ effects, thus the calculation of the Higgs mass is still affected by residual large logarithms (e.g., those controlled by the electroweak gauge couplings).

Of course, the impact of the second and third sources of uncertainty discussed above depends strongly on the considered point in the MSSM parameter space. Higher-order effects in the threshold corrections at the matching scale might become more relevant for non-degenerate SUSY masses or for lower $\tilde{m}$ (where the couplings $g_{t}$ and $g_{3}$ are larger). Also, the effects suppressed by the superparticle masses become obviously larger for lower $\tilde{m}$. In particular, for SUSY mass parameters all equal to $\tilde{m}=1 \mathrm{TeV}$ we can expect the RG resummation to play a lesser role in the accuracy of the Higgs-mass calculation, while the corrections that we neglect become more relevant. Taking again $\tan \beta=20$ and varying $X_{t}$, we find a maximal Higgs mass $M_{h}^{\max } \approx 123 \mathrm{GeV}$ in this scenario (see the "maximal mixing" line on the left edge of figure 1). In contrast, FeynHiggs predicts $M_{h}^{\max } \approx 129-131 \mathrm{GeV}$ (depending on the code's settings), while codes such as SoftSusy [34, 35], SuSpect [36] and SPheno [37, 38], which compute the mass spectrum of the MSSM including the full one-loop and dominant two-loop corrections to the Higgs masses in the $\overline{\mathrm{DR}}$ scheme, ${ }^{10}$ predict $M_{h}^{\max } \approx 124.5-126.5 \mathrm{GeV}$. Such a spread in the Higgs-mass predictions - in a scenario where there is no obvious argument to favor one calculational approach over the others - points to a large theoretical uncertainty, and to the need of improving the calculation with the inclusion of higher-order effects.

\subsection{High-Scale SUSY}

In High-Scale SUSY, all supersymmetric masses lie around the same scale $\tilde{m}$, which can be much larger than the weak scale. The measured Higgs mass $M_{h}^{\exp }=125.15 \pm 0.25 \mathrm{GeV}$ is reproduced in a band of the $(\tilde{m}, \tan \beta)$ plane, as discussed in ref. [6] (see also refs. [16, 20, 39-44]). Here we update the analysis of ref. [6], including our improved calculation of the supersymmetric threshold corrections discussed in section 2.

The left plot in figure 2 shows our updated result. We assume a degenerate spectrum with all superparticle masses set equal to $\tilde{m}$, and plot our prediction for the Higgs mass $M_{h}$ as function of $\tilde{m}$ for $\tan \beta=\{1,2,4,50\}$, varying the soft SUSY-breaking Higgs-stop coupling $A_{t}$ in order to minimize or maximize $M_{h}$. We also plot the uncertainty on the prediction for $M_{h}$ induced by the experimental uncertainty on the SM input parameters $M_{t}$ and $\alpha_{3}\left(M_{Z}\right)$. The plot shows that, even allowing for a $1 \sigma$ reduction in the pole top mass, the measured value of the Higgs mass cannot be reproduced in this simplified scenario if the common SUSY scale $\tilde{m}$ is larger than roughly $3 \times 10^{11} \mathrm{GeV}$. However, this upper bound on $\tilde{m}$ is very sensitive to the top mass and completely evaporates if $M_{t}$ is reduced within its $3 \sigma$ range.

Next, we consider non-degenerate superparticle spectra. Given that superparticle masses are unknown, we randomly scan over them, varying independently the mass

\footnotetext{
${ }^{10}$ While these three codes implement the same corrections to the Higgs masses, they differ in the determination of the running couplings.
} 
High-scale SUSY

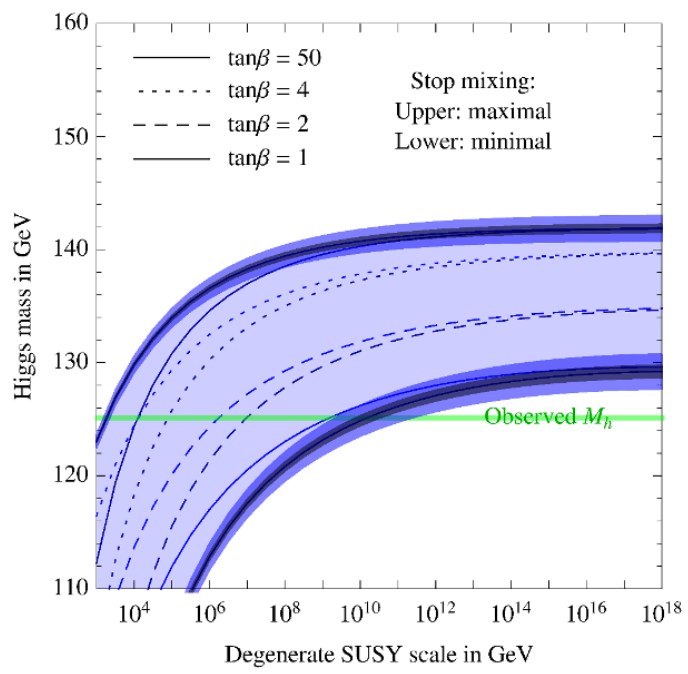

Split-SUSY

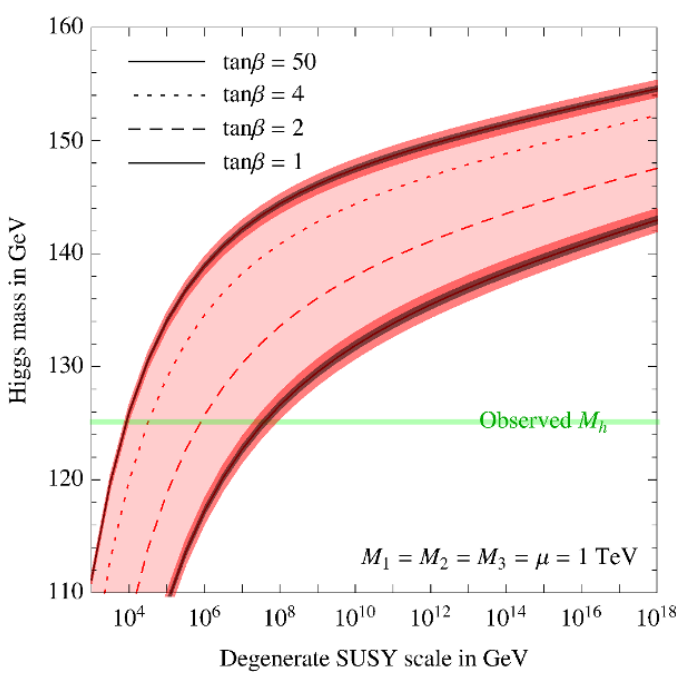

Figure 2. Left: the Higgs mass as a function of the SUSY scale $\tilde{m}$, with a degenerate spectrum of superparticles. We vary the Higgs-stop coupling $A_{t}$ in such a way as to obtain minimal $M_{h}$ (lower lines) and maximal $M_{h}$ (upper lines) at fixed $\tan \beta=\{1,2,4,50\}$. The bands around the extremal solid lines are obtained from $1 \sigma$ variations of $\alpha_{3}\left(M_{Z}\right)$ (thinner band in gray) and $M_{t}$ (larger band in color). The green horizontal band indicates the measured Higgs mass. Right: same as in the left plot, for a split spectrum with gaugino and higgsino masses set to $1 \mathrm{TeV}$ and with $A_{t}=0$.

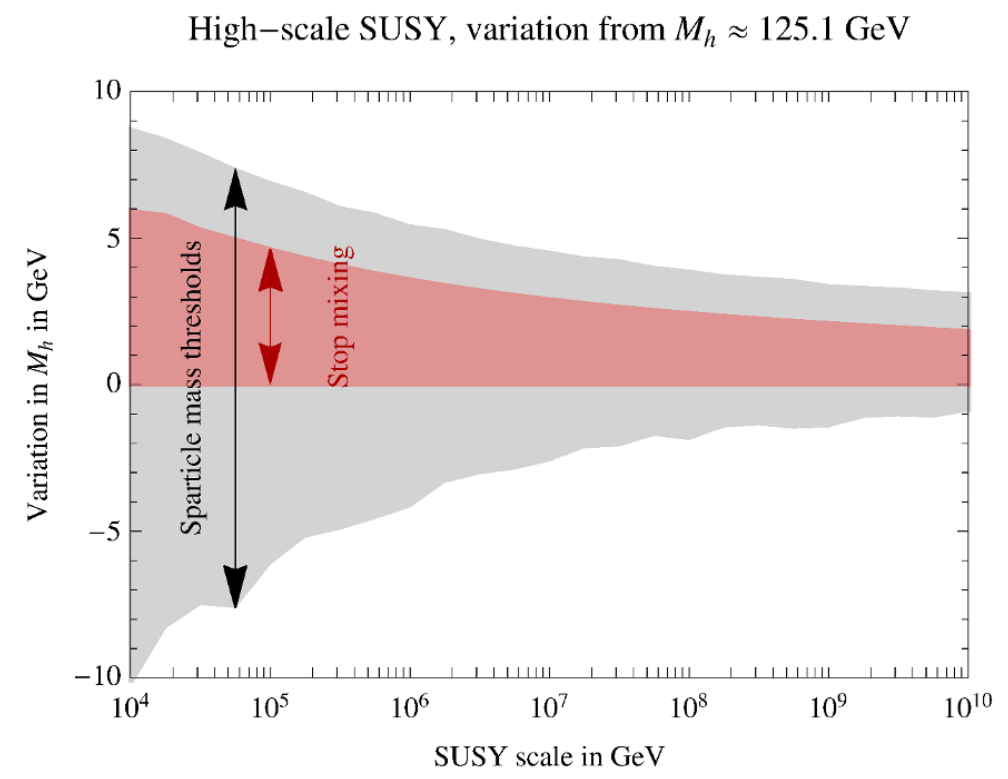

Figure 3. Variation in the prediction for $M_{h}$ in High-Scale SUSY from random scanning each superparticle mass parameter $M_{1}, M_{2}, M_{3}, m_{Q_{i}}, m_{U_{i}}, m_{D_{i}}, m_{E_{i}}, m_{L_{i}}$ and $\mu$ up to a factor 3 above or below the scale $\tilde{m}$, and the Higgs-stop coupling $A_{t}$ within the range allowed by vacuum stability. The darker (red) band shows the variation due only to $A_{t}$. 

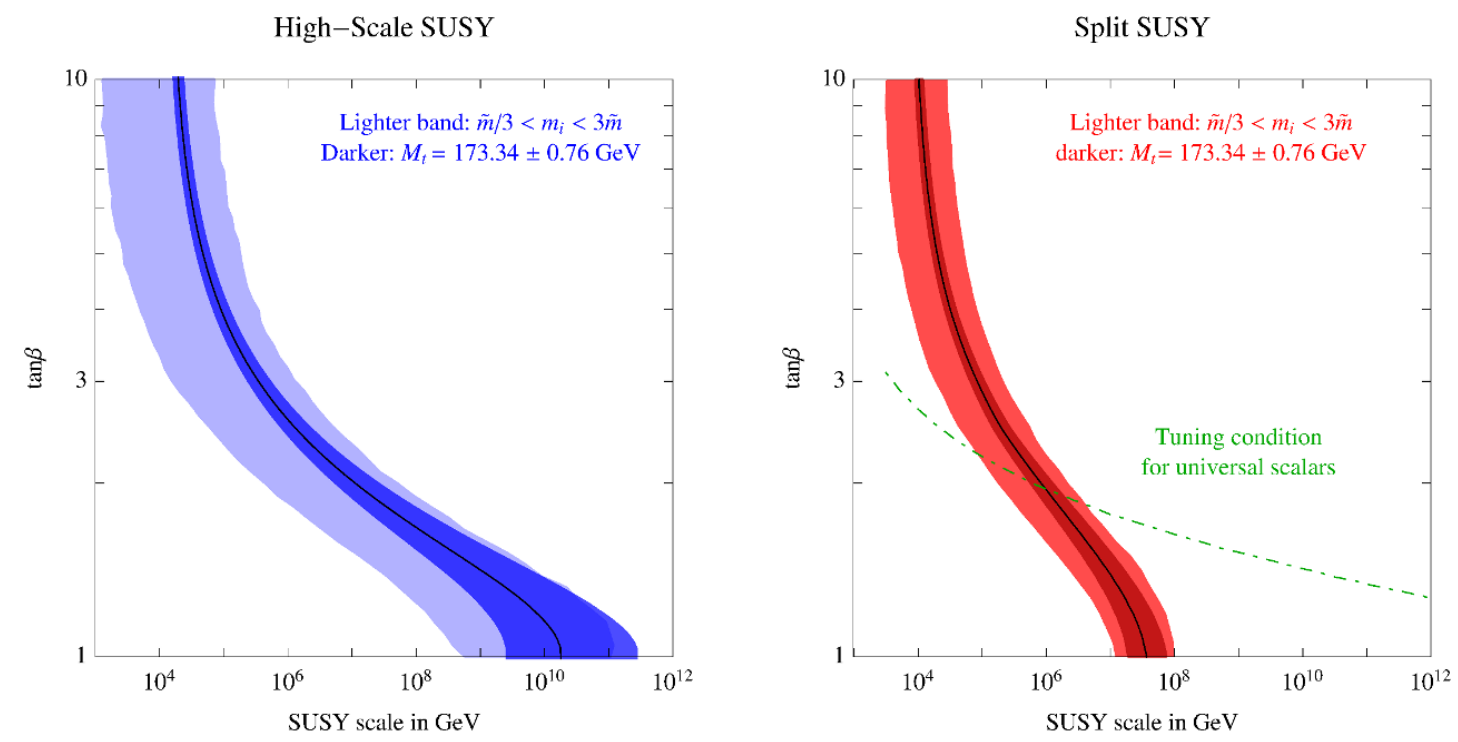

Figure 4. Left: regions in the $(\tilde{m}, \tan \beta)$ plane that reproduce the observed Higgs mass for HighScale SUSY. The black solid line gives the prediction for $X_{t}=0$, mass-degenerate superparticles, and central values for the SM parameters. The light-blue band shows the effect of superparticle thresholds by varying the supersymmetric parameters $M_{1}, M_{2}, M_{3}, m_{Q_{i}}, m_{U_{i}}, m_{D_{i}}, m_{E_{i}}, m_{L_{i}}$ and $\mu$ randomly by up to a factor 3 above or below the scale $\tilde{m}$, and $A_{t}$ within the range allowed by vacuum stability. The dark-blue band corresponds to mass-degenerate superparticles, but includes a $1 \sigma$ variation in $M_{t}$. Right: same as the left plot for the case of Split SUSY. The gaugino and higgsino masses are all set to $1 \mathrm{TeV}$, and $A_{t}=0$. The dot-dashed curve corresponds to the EW tuning condition in the case of universal scalar masses at the GUT scale.

parameters $M_{1}, M_{2}, M_{3}, m_{Q_{i}}, m_{U_{i}}, m_{D_{i}}, m_{E_{i}}, m_{L_{i}}$ (distinguishing the third generation from the other two) and $\mu$ between $\tilde{m} / 3$ and $3 \tilde{m}$, and the Higgs-stop coupling $A_{t}$ within the range allowed by vacuum stability (see next subsection). Figure 3 shows the induced variation in $M_{h}$ with respect to the prediction obtained with degenerate superparticles at a given mass $\tilde{m}$, and with $\tan \beta$ and $A_{t}$ adjusted so that $X_{t}=0$ and $M_{h}=125.15 \mathrm{GeV}$ (this restricts our scan to the range $10^{4} \mathrm{GeV} \lesssim \tilde{m} \lesssim 10^{10} \mathrm{GeV}$, where the measured value of the Higgs mass can be reproduced with central values of the SM input parameters). The darker (red) region in figure 3 denotes the effect of varying only $A_{t}$. The variation in $M_{h}$ is maximal $(\approx 10 \mathrm{GeV})$ in the case of quasi-natural SUSY, $\tilde{m} \approx 10^{4} \mathrm{GeV}$, where $g_{t}$ and $g_{3}$ are large and induce sizable threshold corrections. The variation in $M_{h}$ rapidly decreases with increasing $\tilde{m}$, going down to about $2-3 \mathrm{GeV}$ at the scale $\tilde{m} \approx 10^{10} \mathrm{GeV}$. This shows that the prediction of $M_{h}$ becomes more robust against unknown supersymmetric threshold corrections as one considers larger values of $\tilde{m}$.

Finally, the left plot in figure 4 shows the region in the $(\tilde{m}, \tan \beta)$ plane where the measured Higgs mass is reproduced in High-Scale SUSY. The solid black curve is the prediction obtained with $X_{t}=0$ and exact mass degeneracy at the scale $\tilde{m}$ of all supersymmetric particles, assuming central values of the SM input parameters $M_{h}, M_{t}$ and $\alpha_{3}\left(M_{Z}\right)$. The effect of a $1 \sigma$ variation of the top pole mass is illustrated by the dark blue band. At low $\tan \beta$, corresponding to large $\tilde{m}$, the variation of $M_{t}$ strongly affects $\tilde{m}$. This is mainly because, 
as shown in figure 2, the dependence of $M_{h}$ on $\tilde{m}$ becomes rather flat in High-Scale SUSY when $\tilde{m} \gtrsim 10^{9} \mathrm{GeV}$. Therefore, a small change in $M_{h}$ implies a large change in $\tilde{m}$. The light blue band shows the effect of varying independently all the supersymmetric mass parameters between $\tilde{m} / 3$ and $3 \tilde{m}$, and $A_{t}$ within the range allowed by vacuum stability, as in figure 3. Unlike the case of $M_{t}$, the impact of supersymmetric thresholds in the extraction of $\tilde{m}$ does not show a strong dependence on $\tilde{m}$ itself. This is due an approximate cancellation between two opposing effects: on one hand, as mentioned above, at large $\tilde{m}$ any change in the prediction of $M_{h}$ (whether from $M_{t}$ or from supersymmetric thresholds) has an amplified impact on the determination of $\tilde{m}$. On the other hand, supersymmetric thresholds are smaller at large $\tilde{m}$ (see figure 3 ). The two effects nearly compensate each other, and the impact of supersymmetric thresholds on the light-blue band in figure 4 is fairly uniform.

The left plot in figure 4 shows again how the Higgs mass measurement implies an upper bound on $\tilde{m}$ of about $2 \times 10^{10} \mathrm{GeV}$ in High-Scale SUSY with degenerate supersymmetric masses and central values of the SM parameters. This bound can be raised to about $10^{11} \mathrm{GeV}$ if the supersymmetric square masses differ by about one order of magnitude. So it is difficult for supersymmetric thresholds to raise the bound up to the Planck (or GUT) scale, unless, as previously noticed, $M_{t}$ is $3 \sigma$ below its central value.

As a side remark we note that, in the region of interest, the MSSM top Yukawa coupling $y_{t}$ always remains perturbative. The condition that there are no Landau poles below the Planck scale implies $\tilde{m} \gtrsim 10^{7} \mathrm{GeV}$ for $\tan \beta=1$. This constraint is easily satisfied by the band in the left plot of figure 4 .

Vacuum stability in High-Scale SUSY. Our scans of the SUSY parameter space are restricted to spectra that satisfy the vacuum stability condition. This is an important issue, because this condition eliminates spurious corrections that could reduce the Higgs mass when the parameter $\tilde{X}_{t}=\left(A_{t}-\mu \cot \beta\right)^{2} / m_{Q_{3}} m_{U_{3}}$ is larger than about 12 . The well-known bounds valid in the case of natural SUSY (see, e.g., ref. [45]) need to be adapted to the case of High-Scale SUSY, where the mass term for a combination of the two MSSM Higgs doublets almost vanishes because of the electroweak fine-tuning. In order to determine the upper bound on $\tilde{X}_{t}$, let us consider the scalar potential for the stop-Higgs system

$$
\begin{aligned}
V= & m_{Q_{3}}^{2}\left|\tilde{Q}_{3}\right|^{2}+m_{U_{3}}^{2}\left|\tilde{U}_{3}\right|^{2}+\frac{g_{t}}{\sin \beta}\left(A_{t} H_{u} \tilde{Q}_{3} \tilde{U}_{3}+\mu H_{d}^{*} \tilde{Q}_{3} \tilde{U}_{3}+\text { h.c. }\right) \\
& +\frac{g_{t}^{2}}{\sin ^{2} \beta}\left(\left|H_{u} \tilde{Q}_{3}\right|^{2}+\left|H_{u} \tilde{U}_{3}\right|^{2}+\left|\tilde{Q}_{3} \tilde{U}_{3}\right|^{2}\right)+\text { Higgs-mass terms }+D \text {-terms }
\end{aligned}
$$

where the appropriate $\mathrm{SU}(2)_{L}$ contractions are implicit and where $g_{t}$ is the top Yukawa coupling of the SM. Let us consider the potential along the direction of the approximatelymassless Higgs field $H$ (with $H_{u}=H \sin \beta, H_{d}=\epsilon H^{*} \cos \beta$ ) and along a squark direction such that the $D$-terms vanish. We parameterize this $D$-flat direction with a real field $\phi$, defined by

$$
H_{u}^{i}=\frac{\phi \sin \beta}{\sqrt{2}} \delta_{2}^{i}, \quad H_{d}^{i}=\frac{\phi \cos \beta}{\sqrt{2}} \delta_{1}^{i}, \quad \tilde{Q}_{3}^{i \alpha}=\phi \sqrt{\frac{|\cos 2 \beta|}{2}} \delta_{1}^{i} \delta_{1}^{\alpha}, \quad \tilde{U}_{3}^{\alpha}=\phi \sqrt{\frac{|\cos 2 \beta|}{2}} \delta_{1}^{\alpha},
$$




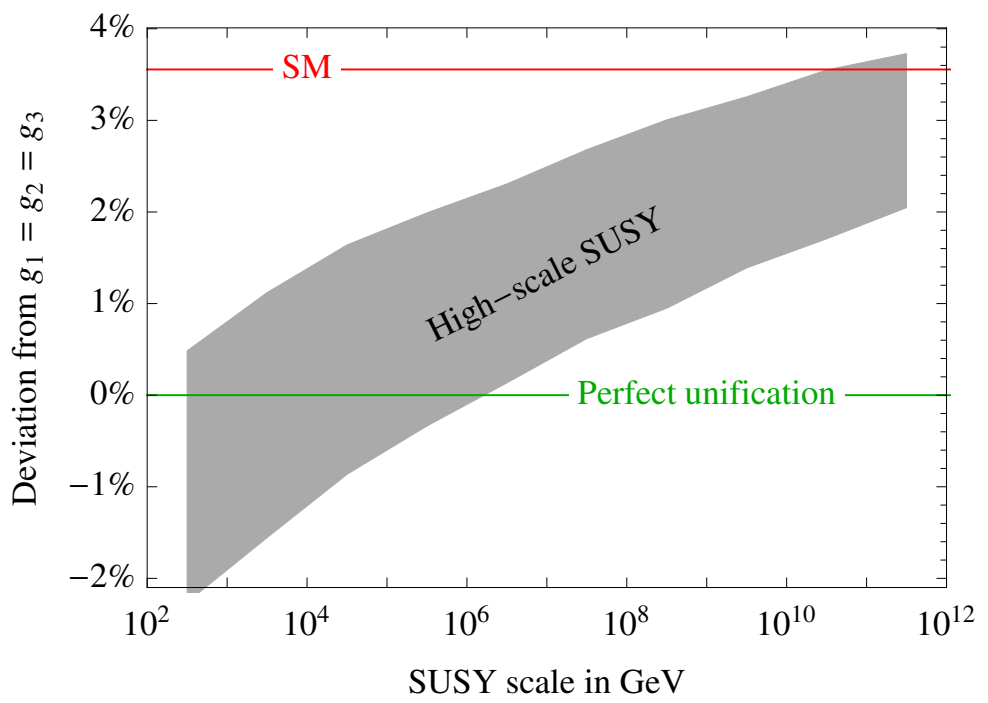

Figure 5. The minimum amount (in percent) by which the unification relation $\hat{g}_{1,2,3}\left(M_{\mathrm{G}}\right)=$ $\hat{g}_{\text {GUT }}$ is missed in High-Scale SUSY. The gray band is obtained by scanning supersymmetric mass parameters by up to a factor 3 above or below the scale $\tilde{m}$, under the condition of reproducing the observed Higgs mass.

where $i$ and $\alpha$ are weak $\mathrm{SU}(2)_{L}$ and color $\mathrm{SU}(3)_{c}$ indices, respectively. The potential for $\phi$ becomes

$$
V=|\cos 2 \beta|\left[\left(m_{Q_{3}}^{2}+m_{U_{3}}^{2}\right) \frac{\phi^{2}}{2}-\frac{g_{t}}{\sqrt{2}}\left(A_{t}-\frac{\mu}{\tan \beta}\right) \phi^{3}+g_{t}^{2}\left(1-\frac{1}{4 \sin ^{2} \beta}\right) \phi^{4}\right] .
$$

The requirement that the color-breaking minimum $\langle\phi\rangle \neq 0$ is not deeper than the electroweak minimum finally implies

$$
\tilde{X}_{t}=\frac{\left(A_{t}-\mu \cot \beta\right)^{2}}{m_{Q_{3}} m_{U_{3}}}<\left(4-\frac{1}{\sin ^{2} \beta}\right)\left(\frac{m_{Q_{3}}}{m_{U_{3}}}+\frac{m_{U_{3}}}{m_{Q_{3}}}\right) .
$$

This constraint has been used to derive the bands in figure 3 and in the left plot of figure 4 .

Gauge-coupling unification. Next, we investigate how supersymmetric threshold corrections affect the GUT condition of gauge-coupling unification in High-Scale SUSY. We employ our full one-loop threshold corrections to the MSSM couplings $\hat{g}_{1}, \hat{g}_{2}, \hat{g}_{3}$ and $\hat{y}_{t}$ in order to compute the values of these couplings at the matching scale $\tilde{m}$. The couplings are then evolved to high energy using the two-loop RGE of the MSSM. In figure 5 we show the minimum amount (in percent) by which one coupling $\hat{g}_{i}\left(M_{\mathrm{G}}\right)$ should be changed in order to achieve an exact crossing $\hat{g}_{1}\left(M_{\mathrm{G}}\right)=\hat{g}_{2}\left(M_{\mathrm{G}}\right)=\hat{g}_{3}\left(M_{\mathrm{G}}\right)$ at some $M_{\mathrm{G}}$, neglecting GUT-scale thresholds. The gray band is obtained by scanning the SUSY mass parameters by up to a factor 3 above or below the scale $\tilde{m}$, and $A_{t}$ within the range allowed by vacuum stability, with $\tan \beta$ adjusted so as to reproduce the measured value of the Higgs mass. For comparison, in the SM $g_{2}\left(M_{\mathrm{G}}\right)$ is larger than the value corresponding to perfect unification by approximately 3.5\%. The figure shows that in High-Scale SUSY perfect gauge-coupling unification can still be achieved as long as the SUSY scale $\tilde{m}$ is lower than a few times $10^{6} \mathrm{GeV}$. 

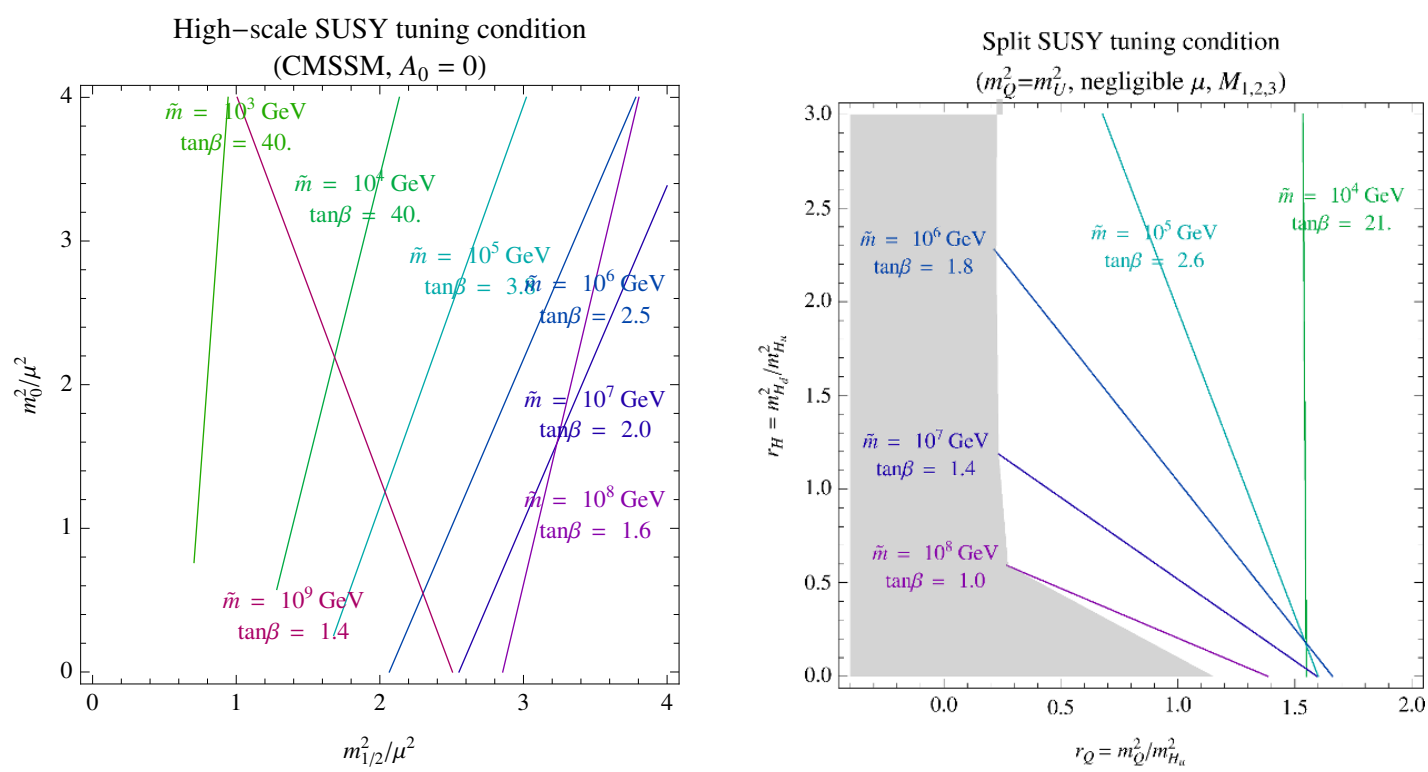

Figure 6. Left: prediction of the SUSY-breaking scale $\tilde{m}$ and the value of $\tan \beta$ from the EW tuning condition and the Higgs mass, in High-Scale SUSY with universal gaugino mass $m_{1 / 2}$ and scalar mass $m_{0}$ at the GUT scale (with $A_{0}=0$ ). The prediction is plotted as a function of the ratios $m_{1 / 2}^{2} / \mu^{2}$ and $m_{0}^{2} / \mu^{2}$ evaluated at the GUT scale. The lines are truncated when the vacuumstability condition is violated. Right: same as in the left plot, in Split SUSY with SU(5) relations for the scalar masses. The prediction is plotted as a function of the ratios $m_{Q}^{2} / m_{H_{u}}^{2}$ and $m_{H_{d}}^{2} / m_{H_{u}}^{2}$ evaluated at the GUT scale. In the shaded region, the EW vacuum is unstable.

Tuning of the EW scale. The measurement of the Higgs mass has been a crucial new element for all schemes of High-Scale SUSY because it provides direct information (although blurred by the unknown parameter $\tan \beta$ ) on the SUSY-breaking scale $\tilde{m}$. Moreover, although such unnatural schemes do not provide any dynamical explanation for the tuning of the EW scale, the very existence of the tuning condition

$$
\tan ^{2} \beta=\left.\frac{m_{H_{d}}^{2}+\mu^{2}}{m_{H_{u}}^{2}+\mu^{2}}\right|_{\tilde{m}}
$$

can teach us something about the pattern of SUSY breaking at energy scales much higher than $\tilde{m}$.

Let us consider a simple pattern of SUSY breaking, in which the superparticle masses at the GUT scale are described by a common gaugino mass $m_{1 / 2}$, a common scalar mass $m_{0}$ and a higgsino mass $\mu$ (for simplicity we set the trilinear coupling $A_{0}=0$ ). For any given value of the ratios $m_{1 / 2} / \mu$ and $m_{0} / \mu$, the measured value of the Higgs mass and the EW tuning condition in eq. (3.5) determine both $\tan \beta$ and the overall scale of supersymmetry breaking, $\tilde{m}$. We show this prediction in the left plot of figure 6 , taking into account the constraint that the vacuum does not break color spontaneously. The fact that solutions are found in most of the plane illustrates the effectiveness of radiative EWSB in supersymmetry. We also note that the quasi-natural solution $\left(\tilde{m}=10^{3} \mathrm{GeV}\right)$ with large $\tan \beta$ corresponds to a nearly vertical line. This is the well-known focus-point behavior, characteristic of low-energy SUSY with universal boundary conditions. The novel result is 
that the model has a second focus point (in which the tuning condition is approximately independent of $\left.m_{0}\right)$ at $\tilde{m}=$ few $\times 10^{8} \mathrm{GeV}$ and low $\tan \beta$.

\section{$3.3 \quad$ Split SUSY}

Another interesting (albeit unnatural) pattern of SUSY breaking is given by Split SUSY [46-48] (see also $[49,50]$ ). The original idea employs two independent mass scales. Scalar masses and $B_{\mu}$ (the mass mixing between the two scalar components of the Higgs superfields) - which correspond to dimension-two, $R$-neutral operators induced by an effective $D$-term supersymmetry breaking — are characterized by the mass parameter $\tilde{m}$. Gaugino/higgsino masses and trilinear couplings $A$ - which correspond to dimensionthree, $R$-charged operators induced by an effective $F$-term supersymmetry breaking - are assumed to be around the weak scale. This spectrum separation can be naturally justified by the different operator dimensionality, by an approximate $R$-symmetry, or by the pattern of supersymmetry breaking. On the other hand, the smallness of the Higgs vacuum expectation value requires a fine-tuning of order $v^{2} / \tilde{m}^{2}$.

We update here the analysis of the Higgs mass in Split SUSY presented in ref. [6], by including our improved calculation of the matching conditions at the scale $\tilde{m}$. The results are shown in the plots on the right of figures 2 and 4, which are the Split-SUSY analogs of the already-described High-Scale SUSY plots on the left of the same figures.

The right plot in figure 2 shows $M_{h}$ as function of the common mass $\tilde{m}$ of a degenerate scalar spectrum. We assume that gauginos and higgsinos are also degenerate with masses $M_{1}=M_{2}=M_{3}=\mu=1 \mathrm{TeV}$, and we show only lines corresponding to $A_{t}=0$ (since in Split SUSY $\left.A_{t} / \tilde{m} \ll 1\right)$.

The right plot in figure 4 shows the allowed region in the $(\tilde{m}, \tan \beta)$ plane. The solid black curve shows again the result in the mass-degenerate case described above. The lightred band shows the broadening of the prediction as the scalar mass parameters are varied between $\tilde{m} / 3$ and $3 \tilde{m}$. Finally, the dark-red band shows the broadening of the prediction of Split SUSY (with universal scalar masses) as $M_{t}$ is varied in its $1 \sigma$ range.

The smallness of $A_{t}$ and $\mu$ in Split SUSY implies that the stop threshold corrections to the Higgs quartic coupling are smaller than in the case of High-Scale SUSY. For this reason, in figure 4 the light-red band is narrower than the light-blue band, for any given $\tan \beta$. Note also that in the case of Split SUSY the uncertainty in $M_{t}$ affects the extraction of $\tilde{m}$ at low $\tan \beta$ less than in High-Scale SUSY (i.e., the dark-red band is narrower than the dark-blue band near $\tan \beta=1$ ). The reason is that the Higgs-mass prediction in Split SUSY (unlike the case of High-Scale SUSY) rises markedly with $\tilde{m}$ in the region around $M_{h}=125 \mathrm{GeV}$.

The LHC data on the Higgs mass rule out the case of large $\tilde{m}$. In particular, $\tilde{m}$ is below $100 \mathrm{TeV}$ if $\tan \beta>4$ and, for $\tan \beta$ close to 1 , it can only reach about $10^{8} \mathrm{GeV}$ (see figure 4). ${ }^{11}$ The determination of $\tilde{m}$ can be translated into a prediction for the gluino lifetime [52]

$$
c \tau_{\tilde{g}}=\left(\frac{2 \mathrm{TeV}}{M_{\tilde{g}}}\right)^{5}\left(\frac{\tilde{m}}{10^{7} \mathrm{GeV}}\right)^{4} 0.4 \mathrm{~m} .
$$

\footnotetext{
${ }^{11}$ See ref. [51] for a modified Split-SUSY scenario in which $\tilde{m}$ can be raised all the way up to the GUT scale.
} 
The Higgs-mass constraint still allows for a wide variety of gluino decay lengths at the LHC. The mean gluino decay length can be larger than the size of the detectors $\left(c \tau_{\tilde{g}} \gtrsim 10 \mathrm{~m}\right)$ for $\tan \beta$ very close to 1 , it is observable as a displaced vertex $\left(c \tau_{\tilde{g}} \gtrsim 50 \mu \mathrm{m}\right)$ for larger $\tan \beta$, while for $\tan \beta \gtrsim 2$ the gluino decays promptly.

It is particularly interesting to consider the implications of the EW tuning condition in the case of Split SUSY $[53,54]$, because the theory at $\tilde{m}$ contains fewer parameters than High-Scale SUSY. The EW tuning condition in eq. (3.5) now becomes

$$
\tan ^{2} \beta=\left.\frac{m_{H_{d}}^{2}}{m_{H_{u}}^{2}}\right|_{\tilde{m}} .
$$

In the simplified case in which the soft scalar masses satisfy SU(5) unification relations at the GUT scale $M_{\mathrm{G}}$, eq. (3.7) can be expressed in terms of the two ratios of masses

$$
r_{H}=\left.\frac{m_{H_{d}}^{2}}{m_{H_{u}}^{2}}\right|_{M_{\mathrm{G}}}, \quad r_{Q}=\left.\frac{m_{Q}^{2}}{m_{H_{u}}^{2}}\right|_{M_{\mathrm{G}}} .
$$

Here $m_{Q}$ denotes the masses of the left and right stop states, which belong to the same irreducible representation of SU(5) and thus are equal at the GUT scale. The EW tuning condition can be expressed as [54]

$$
\tan ^{2} \beta=\frac{\hat{K}+\omega+2 \hat{K} r_{Q}+(1-\omega) r_{H}}{1-\hat{K}-\omega-2 \hat{K} r_{Q}+\omega r_{H}},
$$

where

$$
\begin{aligned}
\hat{K} & =\frac{\sin ^{2} \beta}{2}\left[1-\frac{g_{t}^{2}(\tilde{m})}{g_{t}^{2}\left(M_{\mathrm{G}}\right)}\left(\frac{g_{3}^{2}(\tilde{m})}{g_{3}^{2}\left(M_{\mathrm{G}}\right)}\right)^{-\frac{16}{9}}\left(\frac{g_{2}^{2}(\tilde{m})}{g_{2}^{2}\left(M_{\mathrm{G}}\right)}\right)^{3}\left(\frac{g_{1}^{2}(\tilde{m})}{g_{1}^{2}\left(M_{\mathrm{G}}\right)}\right)^{\frac{13}{99}}\right], \\
\omega & =\frac{1}{22}\left[1-\frac{g_{1}^{2}(\tilde{m})}{g_{1}^{2}\left(M_{\mathrm{G}}\right)}\right] .
\end{aligned}
$$

Note that we have defined $\hat{K}$ in such a way that it is independent of $\tan \beta$ at the leading order. For $\tilde{m}=M_{\mathrm{G}}$ we have $\hat{K}=\omega=0$, while for $\tilde{m}=10 \mathrm{TeV}$ we find $\hat{K}=0.28$ and $\omega=0.024$.

Furthermore, the conditions $m_{Q}^{2}, m_{U}^{2}>0$ (no color-breaking minima) and $m_{H_{u}}^{2}+$ $m_{H_{d}}^{2}>0$ (stability of the EW vacuum) imply the restriction

$$
-\frac{\omega}{1-\omega}<r_{H}<\frac{(4-9 \omega) \tan ^{4} \beta+(7-4 \hat{K} \omega-9 \omega) \tan ^{2} \beta-4 \hat{K} \omega}{3 \omega(1-4 \hat{K}) \tan ^{4} \beta+[-3(1-\omega)+\hat{K}(1-4 \omega)] \tan ^{2} \beta+4(1-\omega) \hat{K}} .
$$

Given the values of $r_{H}$ and $r_{Q}$, the theory predicts both $\tilde{m}$ and $\tan \beta$ from the Higgs mass and the EWSB condition. Our results are shown in the right plot of figure 6 . It is remarkable that acceptable solutions are found in a region close to universality, where both $r_{H}$ and $r_{Q}$ are of order one. A further restriction corresponds to the case $r_{H}=1$, in which there is unification of the Higgs mass parameters, $m_{H_{d}}^{2}=m_{H_{u}}^{2}$, at the GUT scale. Such 
equality is not uncommon in models arising from compactified string theory [42]. Then, the EW tuning conditions in eqs. (3.9) and (3.12) become

$$
r_{Q}=\frac{(1-\hat{K}) \tan ^{2} \beta-1-\hat{K}}{2 \hat{K}\left(1+\tan ^{2} \beta\right)}, \quad \tan ^{2} \beta>\frac{3-4 \hat{K}\left(1+\tan ^{-2} \beta\right)}{3-7 \hat{K}\left(1+\tan ^{-2} \beta\right)} .
$$

The result can be read from the right plot in figure 6 along the horizontal line $r_{H}=1$. The case of exact universality corresponds to the point with $r_{H}=r_{Q}=1$. The EW tuning condition now becomes

$$
\tan ^{2} \beta=\frac{1+3 \hat{K}}{1-3 \hat{K}} .
$$

The prediction for $\tan \beta$ from EW tuning with universal scalars at the GUT scale in eq. (3.14) is shown as a dot-dashed line in the right plot of figure 4 . We find that exact universality corresponds to $\tilde{m} \approx 10^{6} \mathrm{GeV}$ and $\tan \beta \approx 2$, for central values of the SM parameters.

\section{Mini-split with anomaly mediation}

The particular range of values of $\tilde{m}$ determined by the Higgs-mass measurement have fueled interest in a simple version of Split SUSY emerging from anomaly mediation $[55,56]$. The model was originally proposed in ref. [56] and its spectrum was reconsidered in refs. [46-50]. In ref. [57] it was recognized as the simplest model of Split SUSY and its connection with dark matter was elucidated. Subsequent studies are contained in refs. [58-67].

The original motivation of mini-split with anomaly mediation is linked to the observation that gaugino masses require supersymmetry breaking through the $R$-charged $F$-term of a chiral superfield $S$ which must be a singlet under all gauge and global charges

$$
M_{i} \quad \rightarrow \quad \int d^{2} \theta \frac{S}{M_{*}} W_{i \alpha} W_{i}^{\alpha} .
$$

Here $W_{i \alpha}(i=1,2,3)$ is the gauge-strength chiral superfield, and $M_{*}$ is the mediation scale. On the other hand, masses for the scalar components of chiral superfields $Q$ are induced by the $F$-term of any chiral superfield $X$, irrespectively of its global, gauge, or $R$ charges,

$$
\tilde{m}_{Q}^{2} \rightarrow \int d^{4} \theta \frac{X^{\dagger} X}{M_{*}^{2}} Q^{\dagger} Q
$$

This difference becomes important especially in models with dynamical supersymmetry breaking, where no singlets are present. In this case, while scalars acquire a tree-level mass $\tilde{m}=F_{X} / M_{*}$ (where $\sqrt{F_{X}}$ is the scale of supersymmetry breaking, $X=\theta^{2} F_{X}$ ), the leading contribution to a gaugino mass $M_{i}$ comes from one-loop anomaly mediation effects

$$
M_{i}=\frac{\beta_{i}}{g_{i}} m_{3 / 2},
$$

where $g_{i}$ is the corresponding gauge coupling, $\beta_{i}$ its beta function, and $m_{3 / 2}$ is the gravitino mass. 
The same reasoning leads us to conclude that also $A$-terms receive their main contribution from anomaly mediation

$$
A_{y}=-\frac{\beta_{y}}{y} m_{3 / 2}
$$

where $y$ is the corresponding Yukawa coupling and $\beta_{y}$ its beta function.

In order to complete the setup, we have to specify how $\mu$ and $B_{\mu}$ are generated. In general, we expect tree-level contributions to $B_{\mu}$ of order $\tilde{m}^{2}$ induced by

$$
\int d^{4} \theta \frac{X^{\dagger} X}{M_{*}^{2}} H_{u} H_{d},
$$

while $\mu$ is not generated at this level. The most interesting (and plausible) possibility is that $\mu$ is generated by the same mechanism that gives mass to gauginos: gravity [68]. The difference is that, while gaugino masses are generated at one loop, gravity induces the $\mu$ term at tree level. This can be seen by writing the relevant supergravity terms involving the Higgs superfields $\hat{H}_{u, d}$ (normalized such that they have zero canonical dimension) and the conformal compensator $\Phi$, with canonical dimension $d_{\Phi}=1$,

$$
\int d^{4} \theta \Phi^{\dagger} \Phi\left[\hat{H}_{u, d}^{\dagger} \hat{H}_{u, d}+\left(c \hat{H}_{u} \hat{H}_{d}+\text { h.c. }\right)\right]
$$

In terms of the canonical superfields $H_{u, d}=\Phi \hat{H}_{u, d}$, the lagrangian in eq. (4.6) becomes

$$
\int d^{4} \theta\left[H_{u, d}^{\dagger} H_{u, d}+\left(c \frac{\Phi^{\dagger}}{\Phi} H_{u} H_{d}+\text { h.c. }\right)\right]
$$

While usually, because of scale invariance, the dependence on $\Phi$ drops from the classical lagrangian once the latter is expressed in terms of canonically-normalized fields and it reappears only through the scale anomaly in the $\beta$-functions, here we have an explicit dependence on $\Phi$ at tree level. After supersymmetry breaking $\left(\Phi \propto 1-m_{3 / 2} \theta^{2}\right)$, we find

$$
\mu=c m_{3 / 2}, \quad B_{\mu}=c m_{3 / 2}^{2} .
$$

If the coupling constant $c$ is of order one, then the typical mass scale of both $\mu$ and $B_{\mu}$ is the gravitino mass $m_{3 / 2}$, which is parametrically equal to the scalar-mass scale $\tilde{m}$ when the mediation scale $M_{*}$ is close to the Planck scale. However, $c$ is the only PQ-breaking parameter in the theory and it could be small for symmetry reasons. Thus, $\mu$ could take any value between $m_{3 / 2}$ and the weak scale. Irrespectively of the value of $c$, eq. (4.8) implies

$$
B_{\mu}=\mu m_{3 / 2} .
$$

This is an interesting relation because it allows us to link the value of $\mu$ directly to $\tan \beta$. Indeed, after EW breaking, we have $\sin 2 \beta=2\left|B_{\mu}\right| / m_{A}^{2}$, where $m_{A}$ is the Higgs pseudoscalar mass, and thus

$$
\sin 2 \beta=\frac{2|\mu| m_{3 / 2}}{m_{A}^{2}}
$$


The expression of the pseudoscalar mass $m_{A}^{2}=m_{H_{u}}^{2}+m_{H_{d}}^{2}+2 \mu^{2}$ can be simplified with the help of the EW tuning condition eq. (3.5) and becomes

$$
m_{A}^{2}=\left(1+\tan ^{-2} \beta\right)\left(m_{H_{d}}^{2}+\mu^{2}\right) .
$$

Hence, eq. (4.10) can be rewritten as

$$
\tan \beta=\frac{m_{H_{d}}^{2}}{|\mu| m_{3 / 2}}+\frac{|\mu|}{m_{3 / 2}} .
$$

We can now summarize the features of the spectrum of mini-split with anomaly mediation.

(i) Squarks and sleptons: supersymmetric scalars are characterized by the mass scale $\tilde{m}$, although the details of the spectrum are not calculable. The typical size of scalar mass is related to the gravitino mass by $\tilde{m} \approx\left(M_{\mathrm{Pl}} / M_{*}\right) m_{3 / 2}$, where $M_{*}$ is the mediation scale. The requirement that $M_{*}$ is larger than the unification scale, in order not to affect gauge coupling unification, implies $1 \lesssim \tilde{m} / m_{3 / 2} \lesssim 10^{3}$. However, the simplest possibility is that $\tilde{m} / m_{3 / 2}$ is of order one and gravity is the only mediator of supersymmetry breaking.

(ii) Gauginos: anomaly mediation gives precise predictions for the physical masses of the gauginos, in terms of $m_{3 / 2}[69,70]$. In our analysis we include the next-toleading-order corrections controlled by the strong and top-Yukawa couplings, as well as two important effects controlled by the weak gauge couplings. Of the latter, the first consists of logarithms of $\tilde{m} / M_{i}$, which take into account how the gaugino masses deviate from the anomaly-mediation trajectory after squarks and sleptons are integrated out. The second effect is relevant when $\mu$ is larger than the weak scale. In this case, the Higgs superfields act as messengers of supersymmetry breaking and give a one-loop contribution to the gaugino masses proportional to $\mu$. Assuming common mass terms for the squarks and for the sleptons, the physical gaugino masses are

$$
\begin{aligned}
M_{\widetilde{B}}=M_{1}(Q) & {\left[1+\frac{C_{\mu}}{11}+\frac{g_{1}^{2}}{80 \pi^{2}}\left(-\frac{41}{2} \ln \frac{Q^{2}}{M_{1}^{2}}-\frac{1}{2} \ln \frac{\mu^{2}}{M_{1}^{2}}\right.\right.} \\
+ & \left.\left.\ln \frac{m_{A}^{2}}{M_{1}^{2}}+11 \ln \frac{m_{\tilde{q}}^{2}}{M_{1}^{2}}+9 \ln \frac{m_{\tilde{\ell}}^{2}}{M_{1}^{2}}\right)+\frac{g_{3}^{2}}{6 \pi^{2}}-\frac{13 g_{t}^{2}}{264 \pi^{2} \sin ^{2} \beta}\right] \\
M_{\widetilde{W}}=M_{2}(Q) & {\left[1+C_{\mu}+\frac{g_{2}^{2}}{16 \pi^{2}}\left(\frac{19}{6} \ln \frac{Q^{2}}{M_{2}^{2}}-\frac{1}{6} \ln \frac{\mu^{2}}{M_{2}^{2}}\right.\right.} \\
+ & \left.\left.\frac{1}{3} \ln \frac{m_{A}^{2}}{M_{2}^{2}}+3 \ln \frac{m_{\tilde{q}}^{2}}{M_{2}^{2}}+\ln \frac{m_{\tilde{\ell}}^{2}}{M_{2}^{2}}\right)+\frac{3 g_{3}^{2}}{2 \pi^{2}}-\frac{3 g_{t}^{2}}{8 \pi^{2} \sin ^{2} \beta}\right] \\
M_{\tilde{g}}=M_{3}(Q) & {\left[1+\frac{g_{3}^{2}}{16 \pi^{2}}\left(7 \ln \frac{Q^{2}}{M_{3}^{2}}+4 \ln \frac{m_{\tilde{q}}^{2}}{M_{3}^{2}}+\frac{25}{3}-2 F\left(\frac{M_{3}^{2}}{m_{\tilde{q}}^{2}}\right)\right)+\frac{g_{t}^{2}}{12 \pi^{2} \sin ^{2} \beta}\right] }
\end{aligned}
$$

where

$$
M_{1}(Q)=\frac{33 g_{1}^{2}(Q)}{80 \pi^{2}} m_{3 / 2}, \quad M_{2}(Q)=\frac{g_{2}^{2}(Q)}{16 \pi^{2}} m_{3 / 2}, \quad M_{3}(Q)=-\frac{3 g_{3}^{2}(Q)}{16 \pi^{2}} m_{3 / 2},
$$


$g_{i}(Q)$ are the gauge couplings of the SM renormalized in the $\overline{\mathrm{MS}}$ scheme at a generic scale $Q$, and

$$
\begin{aligned}
C_{\mu} & =\frac{\mu}{m_{3 / 2}} \frac{m_{A}^{2} \sin 2 \beta}{m_{A}^{2}-\mu^{2}} \ln \frac{m_{A}^{2}}{\mu^{2}} \\
F(x) & =3\left[\frac{3}{2}-\frac{1}{x}-\left(\frac{1}{x}-1\right)^{2} \ln |1-x|\right]=x+\mathcal{O}\left(x^{2}\right) .
\end{aligned}
$$

(iii) Higgsinos and $\tan \beta$ : the higgsino mass $\mu$ is expected to be of order $m_{3 / 2}$, if there is no suppression related to PQ breaking. Otherwise, $\mu$ is a free parameter, which could vary between $m_{3 / 2}$ and the weak scale. In general, $B_{\mu}$ is of order $\tilde{m}^{2}$ and $\tan \beta$ could take any value. However, when $\mu$ and $B_{\mu}$ are generated by the same operator and eq. (4.9) holds, then $\tan \beta$ is determined according to eq. (4.12).

Mini-split with anomaly mediation has several theoretical and phenomenological attractive features. It retains the positive aspects of Split SUSY (gauge coupling unification, dark matter candidates, easing of the flavor problem) without requiring the artificial (although possible [46, 48, 71]) suppression of one-loop anomaly-mediated gravitational contributions. It retains the positive aspects of anomaly mediation (elegance, predictivity, viability of dynamical supersymmetry breaking) without introducing the problem of tachyonic sleptons [55]. Moreover, the most relevant point for our present analysis is that mini-split with anomaly mediation gives a prediction for the Higgs mass in the right range, as we will show in the next section. Of course the drawback is that the theory is not technically natural.

\subsection{Phenomenology of mini-split with anomaly mediation}

The theory is essentially described in terms of 4 parameters: $\tilde{m}, m_{3 / 2}, \mu$ and $\tan \beta$. One of these parameters is fixed by the value of the Higgs mass, and two more parameters can be fixed under certain assumptions. If gravity is the only mediator of supersymmetry breaking, $\tilde{m}$ is roughly equal to $m_{3 / 2}$. If we assume that the operator in eq. (4.5) is absent and that both $B_{\mu}$ and $\mu$ originate from the operator in eq. (4.6), then the value of $\tan \beta$ is given by eq. (4.12) with $m_{H_{d}}^{2}=\tilde{m}^{2}$.

The scale of supersymmetry breaking in mini-split with anomaly mediation is very favorable for explaining the observed Higgs mass. As an example, figure 7 shows the prediction of the Higgs mass as a function of the gluino mass $M_{\tilde{g}}$ (or, equivalently, of the gravitino mass $m_{3 / 2}$ ), in the special case in which all soft scalar masses are equal to $m_{3 / 2}$. A successful prediction is obtained for $\tan \beta$ in the range between 2 and 3 . We also show the impact of varying $\mu$ between $m_{3 / 2}$ and the gaugino mass scale: the change in the Higgs mass is mild, in the range of $2-3 \mathrm{GeV}$.

In spite of having relatively few free parameters, the theory has a rich variety of possibilities for the nature of the LSP and this has important implications for dark matter. While most studies focused on the case in which $\mu$ is of the order of the gaugino masses, new possibilities for DM appear when $\mu$ is allowed to vary (for some studies of the case 


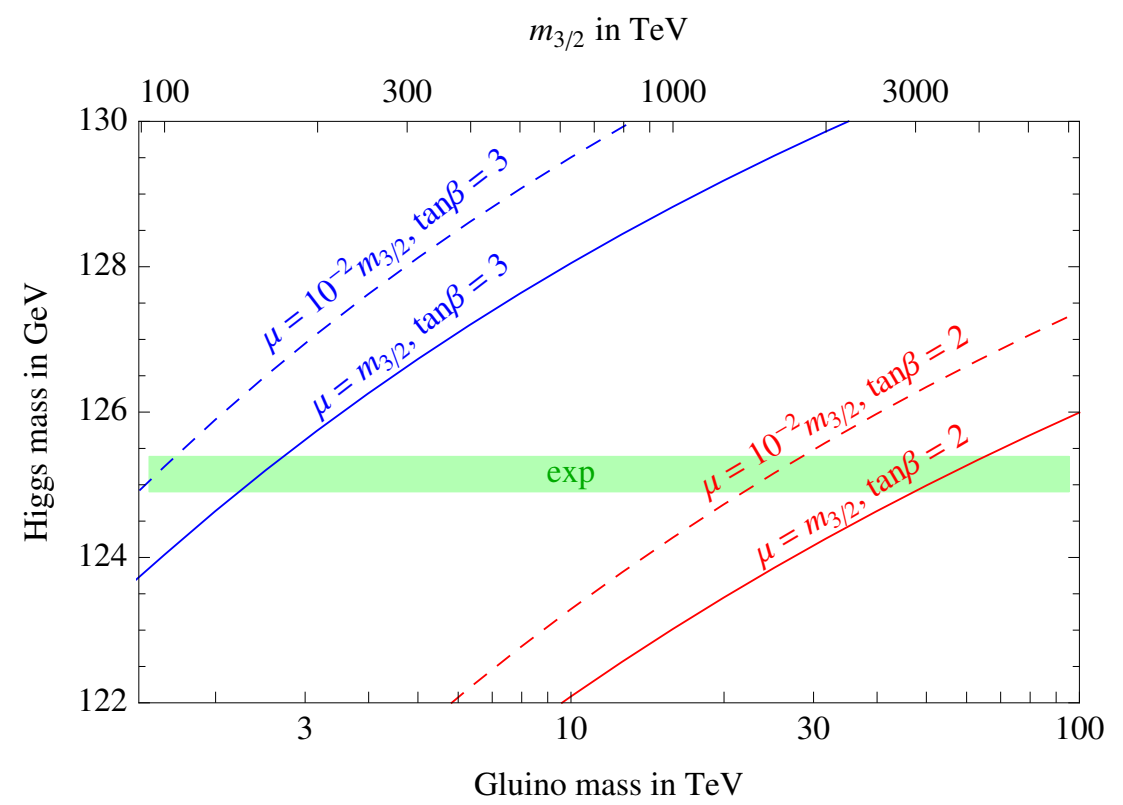

Figure 7. Predicted Higgs mass in mini-split with anomaly mediation, in the case of soft scalar masses equal to $m_{3 / 2}$. The result is shown as a function of the gluino mass $M_{\tilde{g}}$ (or, equivalently, the gravitino mass $m_{3 / 2}$ ) for different values of $\tan \beta$ and of $\mu$.

$\mu=\mathcal{O}\left(m_{3 / 2}\right)$, see refs. [64, 72]). The important parameter that defines the nature of the LSP is $C_{\mu}$, which is defined in eq. (4.15) and describes the source of electroweak gaugino masses coming from the breaking of supersymmetry in the Higgs-higgsino system. In the ordinary case of anomaly mediation with $\mu$ at the weak scale, $C_{\mu}$ is $\mathcal{O}(\alpha / 4 \pi)$, thus its contribution to gaugino masses is parametrically equal to the one-loop corrections in eqs. (4.13a) and (4.13b). However, when $\mu$ is of the same size as $m_{3 / 2}$, the parameter $C_{\mu}$ is of order unity and its contribution to gaugino masses is comparable to the leading effect in anomaly mediation. In the special case in which both $B_{\mu}$ and $\mu$ are generated by eq. (4.6), $C_{\mu}$ becomes a function of a single mass ratio,

$$
\left|C_{\mu}\right|=\frac{2 \ln \left(m_{A}^{2} / \mu^{2}\right)}{m_{A}^{2} / \mu^{2}-1} .
$$

Depending on the value of $C_{\mu}$, the gaugino mass spectrum and the nature of the LSP change, as illustrated in figure 8 , where the three gaugino masses (in units of $m_{3 / 2}$ ) are plotted as functions of $C_{\mu}$. This change in the mass spectrum is important for two reasons. First, different options for the LSP allow for a richer variety of DM candidates with different perspectives for discovery in DM detection experiments. Second, a more compressed gaugino spectrum increases the chances of discovery at the LHC, once the overall mass scale is fixed by DM relic abundance arguments. The various alternatives for the LSP are the following.

- Wino LSP: for $\left|C_{\mu}\right| \lesssim 4$, the LSP is the Wino. This case includes the usual mass spectrum of Split SUSY with $\mu$ at the EW scale. If the Wino is a DM thermal relic, 


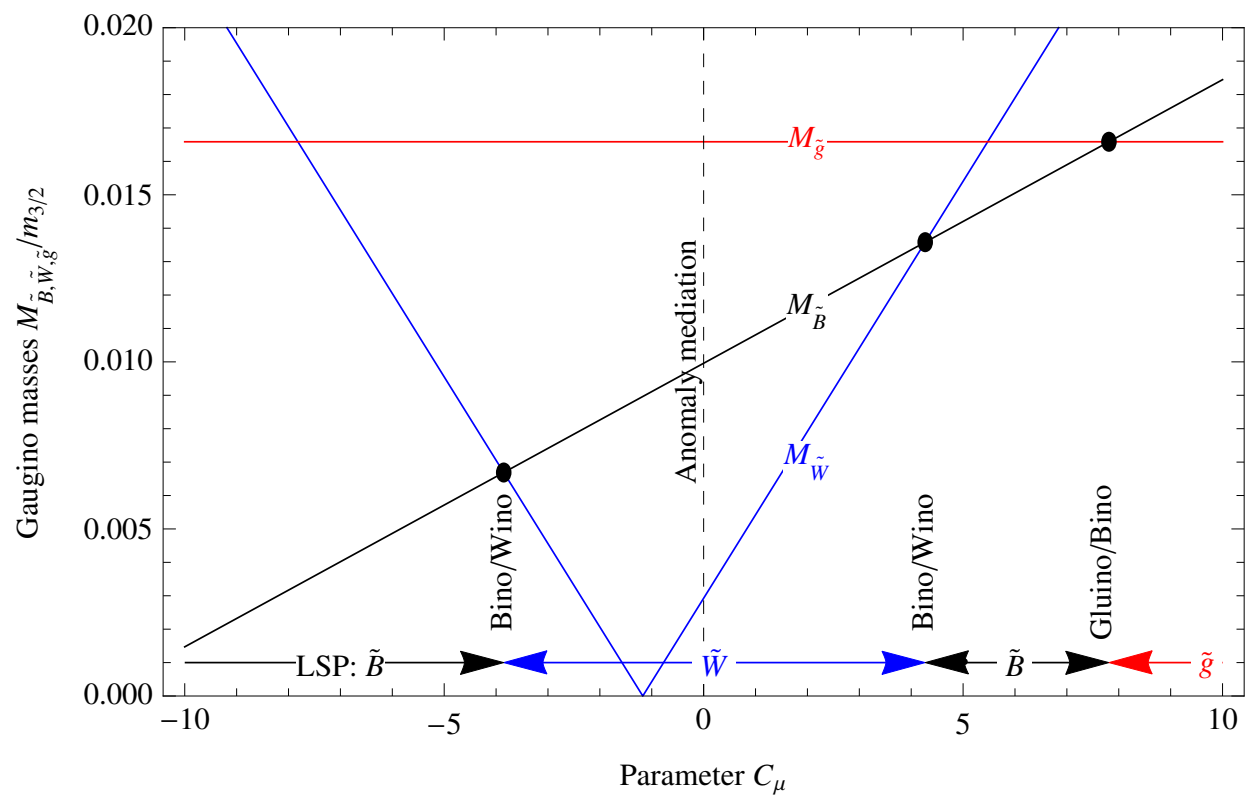

Figure 8. Physical gaugino masses in units of $m_{3 / 2}$ in mini-split with anomaly mediation, as a function of the parameter $C_{\mu}$ defined in eq. (4.15).

then $M_{\widetilde{W}}=2.7 \mathrm{TeV}$. The model is outside the reach of the LHC, even in the most favorable case in which $C_{\mu}$ is in its upper range, and $M_{\tilde{g}} / M_{\widetilde{W}}$ is as small as 1.2. Direct detection of thermal Wino DM is difficult, but the prospects from indirect searches are much more promising. Current bounds from gamma rays are already rather constraining $[73,74]$, although very dependent on the assumptions on the halo profiles.

- Higgsino LSP: for $C_{\mu} \approx 0$, the higgsino can be the LSP. Thermal relic DM is obtained for a higgsino mass of $1.1 \mathrm{TeV}$. This implies the lower bound $M_{\tilde{g}}>6.6 \mathrm{TeV}$. Thus, the gluino is too heavy to be probed at the LHC, in the case of a thermal relic pure higgsino.

- Bino LSP: for $C_{\mu}<-3.9$ and $4.1<C_{\mu}<7.8$, the Bino is the LSP. Its thermal relic abundance would overclose the universe, so Bino DM requires some source of late entropy injection or low reheat temperature $[75,76]$. In the window $4.1<C_{\mu}<7.8$, the gaugino mass spectrum is fairly compressed, with the gluino mass larger than the LSP mass by $20 \%$ or less.

- Gluino LSP: for $C_{\mu}>7.8$, the gluino is the LSP. This case is not acceptable for $\mathrm{DM}$, but it could be interesting for collider searches. The gluino could escape cosmological constraints with the help of small $R$-violating effective interactions that make the LSP unstable. From the collider point of view, the gluino can behave as a stable, unstable, or long-lived particle, depending on the strength of the effective $R$-violation.

- Bino-Wino LSP: for $\left|C_{\mu}\right| \approx 4$, the LSP can be a well-tempered Bino-Wino. For $10 \%$ mass splittings, the mass of the LSP can be in the range of several hundred GeV [57]. 

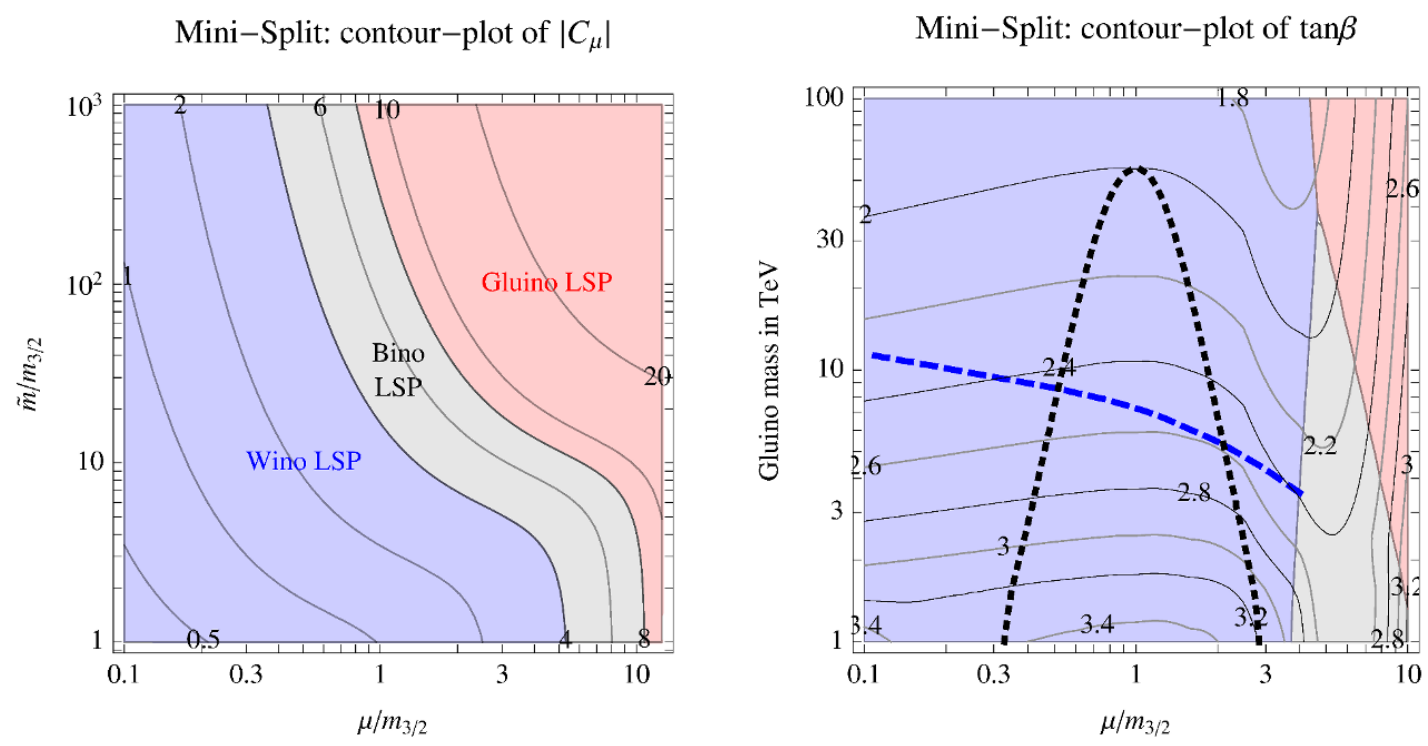

Figure 9. Left: $C_{\mu}$ as a function of the mass ratios $\mu / m_{3 / 2}$ and $\tilde{m} / m_{3 / 2}$. Right: the value of $\tan \beta$ that reproduces the Higgs mass as a function of $\mu / m_{3 / 2}$ and of the physical gluino mass $M_{\tilde{g}}$ for $\tilde{m}=m_{3 / 2}$. Along the dashed blue curve, the Wino thermal DM abundance reproduces the observed DM density. Along the short-dashed black curve $\tan \beta$ as predicted by eq. (4.12) reproduces the observed $M_{h}$. In the blue region, the LSP is the Wino; in the gray region, the LSP is the Bino; in the red region, the LSP can be either the gluino or the Bino, depending on the sign of $\mu$; Higgsino $\mathrm{DM}$ is obtained for $\left|\mu / m_{3 / 2}\right| \lesssim 0.003$.

We find that $M_{\tilde{g}} / M_{\widetilde{W}}=2.4\left(\right.$ for $\left.C_{\mu} \approx-4\right)$ and $M_{\tilde{g}} / M_{\widetilde{W}}=1.2\left(\right.$ for $\left.C_{\mu} \approx 4\right)$. So these cases are particularly favorable for the LHC: the DM particle can be light, the gluino is not much heavier than the LSP, and their mass ratio is precisely determined.

- Higgsino-Wino LSP: for $C_{\mu} \approx 0$, the LSP can be a mixture of higgsino and Wino. Not much is gained in terms of relic abundance, since both the higgsino and the Wino have relatively large annihilation cross sections, but the detection rate in direct DM experiments can be sizable due to Higgs-boson exchange.

- Gluino-Bino LSP: the value $C_{\mu} \approx 7.8$ allows for the unusual possibility of coannihilation between gluino and Bino. This case was recently discussed in ref. [77]. For mass splittings in the $100-150 \mathrm{GeV}$ range, the Bino can be a thermal relic DM and the gluino be within reach of the LHC. However, the experimental search for gluinos is made difficult by their soft decay products. At present, the LHC bound on the gluino mass completely evaporates as soon as the LSP mass is larger than $500-600 \mathrm{GeV}[78,79]$.

It is useful to express $C_{\mu}$ in terms of the original parameters of the model. Using the expression of the pseudoscalar mass in eq. (4.11), we can rewrite eq. (4.15) as

$$
C_{\mu}=\frac{2 \mu \tan \beta}{m_{3 / 2}} \frac{\tilde{m}^{2}+\mu^{2}}{\left(\tan ^{2} \beta+1\right) \tilde{m}^{2}+\mu^{2}} \ln \left[\left(1+\tan ^{-2} \beta\right)\left(1+\frac{\tilde{m}^{2}}{\mu^{2}}\right)\right] .
$$


In the left panel of figure 9 we plot $C_{\mu}$ as a function of the mass ratios $\mu / m_{3 / 2}$ and $\tilde{m} / m_{3 / 2}$, fixing $\tan \beta$ with the requirement of a correct value for the Higgs mass. This shows that values of $\left|C_{\mu}\right|$ in the range 1-10 can be easily obtained for natural choices of the fundamental parameters. Finally, in the right panel of figure 9 we present the map of the various LSP regions in a plane spanned by the physical gluino mass and $\mu / m_{3 / 2}$, under the restrictive assumption of exact universality of scalar masses with $\tilde{m}=m_{3 / 2}$. In this plane we show contours of $\tan \beta$, extracted from the Higgs mass measurement. This figure illustrates once again the mild dependence on $\mu$ of the prediction for $\tan \beta$ (or, equivalently, for the Higgs mass), as $\mu$ is varied from $m_{3 / 2}$ to the gaugino mass scale. The assumption of eq. (4.12) (with $m_{H_{d}}^{2}=\tilde{m}^{2}=m_{3 / 2}^{2}$ ) fixes one extra parameter and constrains the theory to live along the black short-dashed line.

\section{Conclusions}

As collider and DM experiments keep on setting more stringent constraints on low-energy SUSY, the interest is shifting towards models in which supersymmetry is broken at a scale larger than the natural scale $M_{Z}$. In this paper, we have performed a thorough analysis of the Higgs mass in such "unnatural" models.

Our main new computational result is contained in section 2, where we give complete expressions for the one-loop threshold corrections to the Higgs quartic coupling $\lambda$, the top Yukawa coupling, gauge couplings, and gaugino couplings (for Split SUSY) evaluated at the SM/MSSM, SM/Split-SUSY and Split-SUSY/MSSM matching scales. For the Higgs quartic coupling $\lambda$, we include also two-loop QCD threshold corrections. Our results complete and correct previous literature on the subject. Furthermore, we adopt the extraction of SM parameters with NNLO precision, using the results of ref. [4].

In section 3, we applied our results to special realizations of supersymmetry broken at scales larger than $M_{Z}$. The first case refers to quasi-natural SUSY, in which supersymmetric particle masses are in the multi-TeV range. Although the scale of supersymmetry breaking $\tilde{m}$ is not far from the electroweak scale, a precise calculation of the Higgs mass requires resummation of the logarithms of the ratio $\tilde{m} / M_{Z}$. Our results are presented in figure 1 . We find that the Higgs mass measurement implies $\tilde{m} \gtrsim 10 \mathrm{TeV}$ (no stop mixing) and $\tilde{m} \gtrsim 2 \mathrm{TeV}$ (maximal stop mixing), in the case of moderately large $\tan \beta$ and degenerate supersymmetric mass parameters at the scale $\tilde{m}$.

Next, we considered the case of High-Scale $S U S Y$, in which we let $\tilde{m}$ vary arbitrarily. Our predictions for the values of $\tilde{m}$ and $\tan \beta$ determined by the Higgs mass are shown in figures 2 and 4 . We used our calculation of the supersymmetric threshold corrections to show how non-degenerate spectra affect the Higgs mass determination. In particular, we find that the Higgs mass measurement implies $\tilde{m} \lesssim 2 \times 10^{10} \mathrm{GeV}$ (for degenerate supersymmetric particles) and $\tilde{m} \lesssim 10^{11} \mathrm{GeV}$ (for supersymmetric mass parameters larger or smaller than $\tilde{m}$ by a factor of 3 ), and for the central value of the top mass $M_{t}$. If $M_{t}$ is $3 \sigma$ lower than its central value, SUSY up to the Planck scale becomes allowed. Extra Higgs interactions at large energies can change the picture. We have also explored the 
implications of the relation between $\tilde{m}$ and $\tan \beta$ implied by the measured Higgs mass for gauge-coupling unification and for the tuning required to generate the low scale of $M_{Z}$.

We repeated the exercise for Split SUSY, in which scalar supersymmetric particles have masses of order $\tilde{m}$, while fermionic supersymmetric particles lie around the weak scale. Our results, shown in figure 4, indicate that the Higgs mass constrains the scale of Split SUSY $\tilde{m} \lesssim 6 \times 10^{7} \mathrm{GeV}$ (for degenerate scalar supersymmetric particles) and $\tilde{m} \lesssim 10^{8} \mathrm{GeV}$ (for scalar mass parameters larger or smaller than $\tilde{m}$ by a factor of 3 ). Decreasing the top mass by $1 \sigma$ increases the maximal $\tilde{m}$ by a factor of 2 . For universal scalar mass parameters at the GUT scale, we find that the Higgs mass and the tuning condition determine $\tilde{m} \approx 10^{6} \mathrm{GeV}$ and $\tan \beta \approx 2$. This prediction is relaxed as we allow for non-universality of scalar masses at the GUT scale (see figure 6). However, the constraints from EWSB and color conservation select a region of boundary conditions at the GUT scale centered around complete universality.

Section 4 is devoted to the last scenario we considered: mini-split with anomaly mediation. In this case, scalar particles feel supersymmetry breaking at tree level, while gauginos get mass only from one-loop anomaly mediation effects. We have discussed various possibilities for the origin of the higgsino mass $\mu$, which in principle could be anywhere between the gravitino and gaugino masses. Changing $\mu$ in this range has a limited effect on the Higgs mass (see figure 7), but a very important impact on the nature of the LSP. We have found that mini-split with anomaly mediation, in spite of its few free parameters, can lead to a variety of possibilities for the LSP and thus for the DM candidate, as summarized in figure 8. Moreover, it is possible to obtain very compressed spectra for gaugino masses, in which the gluino does not lie far beyond the DM particle, thus increasing the chance of LHC discovery.

\section{Acknowledgments}

This work was supported by the ESF grant MTT8. by the Research Executive Agency (REA) of the European Commission under the Grant Agreement PITN-GA-2010-264564 (LHCPhenoNet), and by French state funds managed by the ANR (ANR-11-IDEX-000402) in the context of the ILP LABEX (ANR-10-LABX-63). P.S. thanks the authors of refs. $[34,35]$ for useful communication about SoftSusy, and A.S. thanks Alexander Knochel for having pointed out a mistake in the expressions of [6] concerning the heavy Higgs loop contribution to the quartic Higgs coupling.

\section{A Loop functions}

The loop functions that describe the stop contribution to the Higgs quartic coupling are:

$$
\begin{aligned}
& \widetilde{F}_{1}(x)=\frac{x \ln x^{2}}{x^{2}-1}, \\
& \widetilde{F}_{2}(x)=\frac{6 x^{2}\left[2-2 x^{2}+\left(1+x^{2}\right) \ln x^{2}\right]}{\left(x^{2}-1\right)^{3}}, \\
& \widetilde{F}_{3}(x)=\frac{2 x\left[5\left(1-x^{2}\right)+\left(1+4 x^{2}\right) \ln x^{2}\right]}{3\left(x^{2}-1\right)^{2}},
\end{aligned}
$$




$$
\begin{aligned}
& \widetilde{F}_{4}(x)=\frac{2 x\left(x^{2}-1-\ln x^{2}\right)}{\left(x^{2}-1\right)^{2}}, \\
& \widetilde{F}_{5}(x)=\frac{3 x\left(1-x^{4}+2 x^{2} \ln x^{2}\right)}{\left(1-x^{2}\right)^{3}} .
\end{aligned}
$$

The extra loop functions that describe the stop contribution to the top Yukawa coupling are:

$$
\begin{aligned}
\widetilde{F}_{6}(x) & =\frac{x^{2}-3}{4\left(1-x^{2}\right)}+\frac{x^{2}\left(x^{2}-2\right)}{2\left(1-x^{2}\right)^{2}} \ln x^{2}, \\
\widetilde{F}_{7}(x) & =\frac{-3\left(x^{4}-6 x^{2}+1\right)}{2\left(x^{2}-1\right)^{2}}+\frac{3 x^{4}\left(x^{2}-3\right)}{\left(x^{2}-1\right)^{3}} \ln x^{2}, \\
\widetilde{F}_{8}\left(x_{1}, x_{2}\right) & =-2+\frac{2}{x_{1}^{2}-x_{2}^{2}}\left(\frac{x_{1}^{4}}{x_{1}^{2}-1} \ln x_{1}^{2}-\frac{x_{2}^{4}}{x_{2}^{2}-1} \ln x_{2}^{2}\right), \\
\widetilde{F}_{9}\left(x_{1}, x_{2}\right) & =\frac{2}{x_{1}^{2}-x_{2}^{2}}\left(\frac{x_{1}^{2}}{x_{1}^{2}-1} \ln x_{1}^{2}-\frac{x_{2}^{2}}{x_{2}^{2}-1} \ln x_{2}^{2}\right) .
\end{aligned}
$$

Finally, the loop functions for the gaugino-higgsino corrections to both $\lambda$ and $g_{t}$ are [6]

$$
\begin{aligned}
f(r) & =\widetilde{F}_{5}(r), \quad g(r)=\widetilde{F}_{7}(r), \\
f_{1}(r) & =\frac{6\left(r^{2}+3\right) r^{2}}{7\left(r^{2}-1\right)^{2}}+\frac{6\left(r^{2}-5\right) r^{4} \ln r^{2}}{7\left(r^{2}-1\right)^{3}} \\
f_{2}(r) & =\frac{2\left(r^{2}+11\right) r^{2}}{9\left(r^{2}-1\right)^{2}}+\frac{2\left(5 r^{2}-17\right) r^{4} \ln r^{2}}{9\left(r^{2}-1\right)^{3}}, \\
f_{3}(r) & =\frac{2\left(r^{4}+9 r^{2}+2\right)}{3\left(r^{2}-1\right)^{2}}+\frac{2\left(r^{4}-7 r^{2}-6\right) r^{2} \ln r^{2}}{3\left(r^{2}-1\right)^{3}} \\
f_{4}(r) & =\frac{2\left(5 r^{4}+25 r^{2}+6\right)}{7\left(r^{2}-1\right)^{2}}+\frac{2\left(r^{4}-19 r^{2}-18\right) r^{2} \ln r^{2}}{7\left(r^{2}-1\right)^{3}} \\
\frac{4}{3} f_{5}\left(r_{1}, r_{2}\right) & =\frac{1+\left(r_{1}+r_{2}\right)^{2}-r_{1}^{2} r_{2}^{2}}{\left(r_{1}^{2}-1\right)\left(r_{2}^{2}-1\right)}+\frac{r_{1}^{3}\left(r_{1}^{2}+1\right) \ln r_{1}^{2}}{\left(r_{1}^{2}-1\right)^{2}\left(r_{1}-r_{2}\right)}-\frac{r_{2}^{3}\left(r_{2}^{2}+1\right) \ln r_{2}^{2}}{\left(r_{1}-r_{2}\right)\left(r_{2}^{2}-1\right)^{2}} \\
\frac{7}{6} f_{6}\left(r_{1}, r_{2}\right) & =\frac{r_{1}^{2}+r_{2}^{2}+r_{1} r_{2}-r_{1}^{2} r_{2}^{2}}{\left(r_{1}^{2}-1\right)\left(r_{2}^{2}-1\right)}+\frac{r_{1}^{5} \ln r_{1}^{2}}{\left(r_{1}^{2}-1\right)^{2}\left(r_{1}-r_{2}\right)}-\frac{r_{2}^{5} \ln r_{2}^{2}}{\left(r_{1}-r_{2}\right)\left(r_{2}^{2}-1\right)^{2}} \\
\frac{1}{6} f_{7}\left(r_{1}, r_{2}\right) & =\frac{1+r_{1} r_{2}}{\left(r_{1}^{2}-1\right)\left(r_{2}^{2}-1\right)}+\frac{r_{1}^{3} \ln r_{1}^{2}}{\left(r_{1}^{2}-1\right)^{2}\left(r_{1}-r_{2}\right)}-\frac{r_{2}^{3} \ln r_{2}^{2}}{\left(r_{1}-r_{2}\right)\left(r_{2}^{2}-1\right)^{2}} \\
\frac{2}{3} f_{8}\left(r_{1}, r_{2}\right) & =\frac{r_{1}+r_{2}}{\left(r_{1}^{2}-1\right)\left(r_{2}^{2}-1\right)}+\frac{r_{1}^{4} \ln r_{1}^{2}}{\left(r_{1}^{2}-1\right)^{2}\left(r_{1}-r_{2}\right)}-\frac{r_{2}^{4} \ln r_{2}^{2}}{\left(r_{1}-r_{2}\right)\left(r_{2}^{2}-1\right)^{2}}
\end{aligned}
$$

All functions in eqs. (A.1a)-(A.3a) are equal to 1 when they arguments approach unity, with the exception of $\widetilde{F}_{6}$ which tends to 0 .

Open Access. This article is distributed under the terms of the Creative Commons Attribution License (CC-BY 4.0), which permits any use, distribution and reproduction in any medium, provided the original author(s) and source are credited. 


\section{References}

[1] CMS collaboration, Measurement of the properties of a Higgs boson in the four-lepton final state, Phys. Rev. D 89 (2014) 092007 [arXiv: 1312.5353] [InSPIRE].

[2] CMS collaboration, Observation of the diphoton decay of the Higgs boson and measurement of its properties, arXiv: 1407.0558 [INSPIRE].

[3] ATLAS collaboration, Measurement of the Higgs boson mass from the $H \rightarrow \gamma \gamma$ and $H \rightarrow Z Z^{*} \rightarrow 4 \ell$ channels with the ATLAS detector using $25 \mathrm{fb}^{-1}$ of pp collision data, arXiv: 1406.3827 [INSPIRE].

[4] D. Buttazzo et al., Investigating the near-criticality of the Higgs boson, JHEP 12 (2013) 089 [arXiv: 1307.3536] [INSPIRE].

[5] N. Bernal, A. Djouadi and P. Slavich, The MSSM with heavy scalars, JHEP 07 (2007) 016 [arXiv:0705.1496] [INSPIRE].

[6] G.F. Giudice and A. Strumia, Probing high-scale and split supersymmetry with Higgs mass measurements, Nucl. Phys. B 858 (2012) 63 [arXiv:1108.6077] [InSPIRE].

[7] S.P. Martin and M.T. Vaughn, Regularization dependence of running couplings in softly broken supersymmetry, Phys. Lett. B 318 (1993) 331 [hep-ph/9308222] [INSPIRE].

[8] A. Pilaftsis, Resonant CP-violation induced by particle mixing in transition amplitudes, Nucl. Phys. B 504 (1997) 61 [hep-ph/9702393] [INSPIRE].

[9] Y. Yamada, Gauge dependence of the on-shell renormalized mixing matrices, Phys. Rev. D 64 (2001) 036008 [hep-ph/0103046] [INSPIRE].

[10] J.R. Espinosa and I. Navarro, Scale independent mixing angles, Phys. Rev. D 66 (2002) 016004 [hep-ph/0109126] [INSPIRE].

[11] M. Binger, Higgs boson mass in split supersymmetry at two-loops, Phys. Rev. D 73 (2006) 095001 [hep-ph/0408240] [INSPIRE].

[12] P.P. Giardino and P. Lodone, Threshold corrections to hard supersymmetric relations, Mod. Phys. Lett. A 29 (2014) 1450101 [arXiv:1112.2635] [INSPIRE].

[13] G. Degrassi, P. Slavich and F. Zwirner, On the neutral Higgs boson masses in the MSSM for arbitrary stop mixing, Nucl. Phys. B 611 (2001) 403 [hep-ph/0105096] [INSPIRE].

[14] G. Degrassi and P. Slavich, On the radiative corrections to the neutral Higgs boson masses in the NMSSM, Nucl. Phys. B 825 (2010) 119 [arXiv:0907.4682] [INSPIRE].

[15] M. Brucherseifer, R. Gavin and M. Spira, MSSM Higgs self-couplings: two-loop $\mathcal{O}\left(\alpha_{t} \alpha_{s}\right)$ corrections, arXiv:1309.3140 [INSPIRE].

[16] G. Degrassi et al., Higgs mass and vacuum stability in the standard model at NNLO, JHEP 08 (2012) 098 [arXiv: 1205.6497] [INSPIRE].

[17] J.R. Espinosa and R.-J. Zhang, MSSM lightest CP even Higgs boson mass to $\mathcal{O}\left(\alpha_{s} \alpha_{t}\right)$ : the effective potential approach, JHEP 03 (2000) 026 [hep-ph/9912236] [INSPIRE].

[18] ATLAS, CDF, CMS and D0 collaborations, First combination of Tevatron and LHC measurements of the top-quark mass, arXiv:1403.4427 [INSPIRE].

[19] S. Bethke, The 2009 world average of $\alpha_{s}$, Eur. Phys. J. C 64 (2009) 689 [arXiv:0908.1135] [INSPIRE]. 
[20] P. Draper, G. Lee and C.E.M. Wagner, Precise estimates of the Higgs mass in heavy SUSY, Phys. Rev. D 89 (2014) 055023 [arXiv: 1312.5743] [INSPIRE].

[21] T. Hahn, S. Heinemeyer, W. Hollik, H. Rzehak and G. Weiglein, High-precision predictions for the light CP-even Higgs boson mass of the MSSM, Phys. Rev. Lett. 112 (2014) 141801 [arXiv:1312.4937] [INSPIRE].

[22] J.L. Feng, P. Kant, S. Profumo and D. Sanford, Three-loop corrections to the Higgs boson mass and implications for supersymmetry at the LHC, Phys. Rev. Lett. 111 (2013) 131802 [arXiv: 1306.2318] [INSPIRE].

[23] S. Heinemeyer, W. Hollik and G. Weiglein, FeynHiggs: a program for the calculation of the masses of the neutral CP even Higgs bosons in the MSSM, Comput. Phys. Commun. 124 (2000) 76 [hep-ph/9812320] [INSPIRE].

[24] M. Frank et al., The Higgs boson masses and mixings of the complex MSSM in the Feynman-diagrammatic approach, JHEP 02 (2007) 047 [hep-ph/0611326] [INSPIRE].

[25] S. Heinemeyer, W. Hollik and G. Weiglein, The masses of the neutral CP-even Higgs bosons in the MSSM: accurate analysis at the two loop level, Eur. Phys. J. C 9 (1999) 343 [hep-ph/9812472] [INSPIRE].

[26] A. Brignole, G. Degrassi, P. Slavich and F. Zwirner, On the $\mathcal{O}\left(\alpha_{t}^{2}\right)$ two loop corrections to the neutral Higgs boson masses in the MSSM, Nucl. Phys. B 631 (2002) 195 [hep-ph/0112177] [INSPIRE].

[27] A. Brignole, G. Degrassi, P. Slavich and F. Zwirner, On the two loop sbottom corrections to the neutral Higgs boson masses in the MSSM, Nucl. Phys. B 643 (2002) 79 [hep-ph/0206101] [INSPIRE].

[28] A. Dedes, G. Degrassi and P. Slavich, On the two loop Yukawa corrections to the MSSM Higgs boson masses at large $\tan \beta$, Nucl. Phys. B 672 (2003) 144 [hep-ph/0305127] [INSPIRE].

[29] S. Heinemeyer, W. Hollik, H. Rzehak and G. Weiglein, High-precision predictions for the MSSM Higgs sector at $\mathcal{O}\left(\alpha_{b} \alpha_{s}\right)$, Eur. Phys. J. C 39 (2005) 465 [hep-ph/0411114] [INSPIRE].

[30] S. Heinemeyer, W. Hollik, H. Rzehak and G. Weiglein, The Higgs sector of the complex MSSM at two-loop order: QCD contributions, Phys. Lett. B 652 (2007) 300 [arXiv: 0705. 0746] [inSPIRE].

[31] J.R. Espinosa and R.-J. Zhang, Complete two loop dominant corrections to the mass of the lightest CP even Higgs boson in the minimal supersymmetric standard model, Nucl. Phys. B 586 (2000) 3 [hep-ph/0003246] [INSPIRE].

[32] G. Degrassi, S. Heinemeyer, W. Hollik, P. Slavich and G. Weiglein, Towards high precision predictions for the MSSM Higgs sector, Eur. Phys. J. C 28 (2003) 133 [hep-ph/0212020] [INSPIRE].

[33] B.C. Allanach, A. Djouadi, J.L. Kneur, W. Porod and P. Slavich, Precise determination of the neutral Higgs boson masses in the MSSM, JHEP 09 (2004) 044 [hep-ph/0406166] [INSPIRE].

[34] B.C. Allanach, SOFTSUSY: a program for calculating supersymmetric spectra, Comput. Phys. Commun. 143 (2002) 305 [hep-ph/0104145] [INSPIRE]. 
[35] B.C. Allanach, A. Bednyakov and R. Ruiz de Austri, Higher order corrections, the Higgs mass and unification in the minimal supersymmetric standard model: SOFTSUSY3.5.0, arXiv: 1407.6130 [INSPIRE].

[36] A. Djouadi, J.-L. Kneur and G. Moultaka, SuSpect: a fortran code for the supersymmetric and Higgs particle spectrum in the MSSM, Comput. Phys. Commun. 176 (2007) 426 [hep-ph/0211331] [INSPIRE].

[37] W. Porod, SPheno, a program for calculating supersymmetric spectra, SUSY particle decays and SUSY particle production at $e^{+} e^{-}$colliders, Comput. Phys. Commun. 153 (2003) 275 [hep-ph/0301101] [INSPIRE].

[38] W. Porod and F. Staub, SPheno 3.1: extensions including flavour, CP-phases and models beyond the MSSM, Comput. Phys. Commun. 183 (2012) 2458 [arXiv:1104.1573] [InSPIRE].

[39] L.J. Hall and Y. Nomura, A finely-predicted Higgs boson mass from a finely-tuned weak scale, JHEP 03 (2010) 076 [arXiv:0910.2235] [INSPIRE].

[40] M.E. Cabrera, J.A. Casas and A. Delgado, Upper bounds on superpartner masses from upper bounds on the Higgs boson mass, Phys. Rev. Lett. 108 (2012) 021802 [arXiv:1108.3867] [INSPIRE].

[41] A. Arbey, M. Battaglia, A. Djouadi, F. Mahmoudi and J. Quevillon, Implications of a 125 GeV Higgs for supersymmetric models, Phys. Lett. B 708 (2012) 162 [arXiv:1112.3028] [INSPIRE].

[42] L.E. Ibáñez and I. Valenzuela, The Higgs mass as a signature of heavy SUSY, JHEP 05 (2013) 064 [arXiv:1301.5167] [INSPIRE].

[43] A. Hebecker, A.K. Knochel and T. Weigand, The Higgs mass from a string-theoretic perspective, Nucl. Phys. B $\mathbf{8 7 4}$ (2013) 1 [arXiv:1304.2767] [INSPIRE].

[44] A. Delgado, M. Garcia and M. Quirós, Electroweak and supersymmetry breaking from the Higgs discovery, Phys. Rev. D 90 (2014) 015016 [arXiv: 1312.3235] [INSPIRE].

[45] J.A. Casas, A. Lleyda and C. Muñoz, Strong constraints on the parameter space of the MSSM from charge and color breaking minima, Nucl. Phys. B 471 (1996) 3 [hep-ph/9507294] [INSPIRE].

[46] N. Arkani-Hamed and S. Dimopoulos, Supersymmetric unification without low energy supersymmetry and signatures for fine-tuning at the LHC, JHEP 06 (2005) 073 [hep-th/0405159] [INSPIRE].

[47] G.F. Giudice and A. Romanino, Split supersymmetry, Nucl. Phys. B 699 (2004) 65 [Erratum ibid. B 706 (2005) 65] [hep-ph/0406088] [INSPIRE].

[48] N. Arkani-Hamed, S. Dimopoulos, G.F. Giudice and A. Romanino, Aspects of split supersymmetry, Nucl. Phys. B 709 (2005) 3 [hep-ph/0409232] [INSPIRE].

[49] J.D. Wells, Implications of supersymmetry breaking with a little hierarchy between gauginos and scalars, hep-ph/0306127 [INSPIRE].

[50] J.D. Wells, PeV-scale supersymmetry, Phys. Rev. D 71 (2005) 015013 [hep-ph/0411041] [INSPIRE].

[51] K. Benakli, L. Darmé, M.D. Goodsell and P. Slavich, A fake split supersymmetry model for the $126 \mathrm{GeV}$ Higgs, JHEP 05 (2014) 113 [arXiv: 1312.5220] [INSPIRE]. 
[52] P. Gambino, G.F. Giudice and P. Slavich, Gluino decays in split supersymmetry, Nucl. Phys. B 726 (2005) 35 [hep-ph/0506214] [INSPIRE].

[53] A. Ibarra, Tachyonic squarks in split supersymmetry, Phys. Lett. B 620 (2005) 164 [hep-ph/0503160] [INSPIRE].

[54] A. Delgado and G.F. Giudice, On the tuning condition of split supersymmetry, Phys. Lett. B 627 (2005) 155 [hep-ph/0506217] [INSPIRE].

[55] L. Randall and R. Sundrum, Out of this world supersymmetry breaking, Nucl. Phys. B 557 (1999) 79 [hep-th/9810155] [INSPIRE].

[56] G.F. Giudice, M.A. Luty, H. Murayama and R. Rattazzi, Gaugino mass without singlets, JHEP 12 (1998) 027 [hep-ph/9810442] [INSPIRE].

[57] N. Arkani-Hamed, A. Delgado and G.F. Giudice, The well-tempered neutralino, Nucl. Phys. B 741 (2006) 108 [hep-ph/0601041] [INSPIRE].

[58] L.J. Hall and Y. Nomura, Spread supersymmetry, JHEP 01 (2012) 082 [arXiv:1111.4519] [INSPIRE].

[59] G. Kane, P. Kumar, R. Lu and B. Zheng, Higgs mass prediction for realistic string/M theory vacua, Phys. Rev. D 85 (2012) 075026 [arXiv:1112.1059] [inSPIRE].

[60] B.S. Acharya, G. Kane and P. Kumar, Compactified string theories - generic predictions for particle physics, Int. J. Mod. Phys. A 27 (2012) 1230012 [arXiv:1204.2795] [InSPIRE].

[61] B. Bhattacherjee, B. Feldstein, M. Ibe, S. Matsumoto and T.T. Yanagida, Pure gravity mediation of supersymmetry breaking at the LHC, Phys. Rev. D 87 (2013) 015028 [arXiv: 1207.5453] [INSPIRE].

[62] A. Arvanitaki, N. Craig, S. Dimopoulos and G. Villadoro, Mini-split, JHEP 02 (2013) 126 [arXiv: 1210.0555] [INSPIRE].

[63] L.J. Hall, Y. Nomura and S. Shirai, Spread supersymmetry with wino LSP: gluino and dark matter signals, JHEP 01 (2013) 036 [arXiv:1210.2395] [INSPIRE].

[64] N. Arkani-Hamed, A. Gupta, D.E. Kaplan, N. Weiner and T. Zorawski, Simply unnatural supersymmetry, arXiv:1212.6971 [INSPIRE].

[65] T. Moroi and M. Nagai, Probing supersymmetric model with heavy sfermions using leptonic flavor and CP-violations, Phys. Lett. B 723 (2013) 107 [arXiv:1303.0668] [INSPIRE].

[66] D. McKeen, M. Pospelov and A. Ritz, Electric dipole moment signatures of PeV-scale superpartners, Phys. Rev. D 87 (2013) 113002 [arXiv: 1303.1172] [INSPIRE].

[67] Y. Kahn, M. McCullough and J. Thaler, Auxiliary gauge mediation: a new route to mini-split supersymmetry, JHEP 11 (2013) 161 [arXiv:1308.3490] [INSPIRE].

[68] G.F. Giudice and A. Masiero, A natural solution to the $\mu$ problem in supergravity theories, Phys. Lett. B 206 (1988) 480 [INSPIRE].

[69] T. Gherghetta, G.F. Giudice and J.D. Wells, Phenomenological consequences of supersymmetry with anomaly induced masses, Nucl. Phys. B 559 (1999) 27 [hep-ph/9904378] [INSPIRE].

[70] A. Gupta, D.E. Kaplan and T. Zorawski, Gaugomaly mediation revisited, JHEP 11 (2013) 149 [arXiv:1212.6969] [INSPIRE]. 
[71] M.A. Luty, Weak scale supersymmetry without weak scale supergravity, Phys. Rev. Lett. 89 (2002) 141801 [hep-th/0205077] [INSPIRE].

[72] K. Harigaya, K. Kaneta and S. Matsumoto, Gaugino coannihilations, Phys. Rev. D 89 (2014) 115021 [arXiv:1403.0715] [INSPIRE].

[73] T. Cohen, M. Lisanti, A. Pierce and T.R. Slatyer, Wino dark matter under siege, JCAP 10 (2013) 061 [arXiv:1307.4082] [INSPIRE].

[74] J. Fan and M. Reece, In wino veritas? Indirect searches shed light on neutralino dark matter, JHEP 10 (2013) 124 [arXiv: 1307.4400] [INSPIRE].

[75] G.F. Giudice, E.W. Kolb and A. Riotto, Largest temperature of the radiation era and its cosmological implications, Phys. Rev. D 64 (2001) 023508 [hep-ph/0005123] [INSPIRE].

[76] G.B. Gelmini and P. Gondolo, Neutralino with the right cold dark matter abundance in (almost) any supersymmetric model, Phys. Rev. D 74 (2006) 023510 [hep-ph/0602230] [INSPIRE].

[77] A. De Simone, G.F. Giudice and A. Strumia, Benchmarks for dark matter searches at the LHC, JHEP 06 (2014) 081 [arXiv: 1402.6287] [INSPIRE].

[78] CMS collaboration, Search for new physics in the multijet and missing transverse momentum final state in proton-proton collisions at $\sqrt{s}=8 \mathrm{TeV}$, JHEP 06 (2014) 055 [arXiv: 1402 .4770] [INSPIRE].

[79] ATLAS collaboration, Search for squarks and gluinos with the ATLAS detector in final states with jets and missing transverse momentum using $\sqrt{s}=8$ TeV proton-proton collision data, arXiv: 1405.7875 [INSPIRE]. 